\title{
Forty years of improvements in European air quality: regional policy- industry interactions with global impacts
}

Monica Crippa et al.

Correspondence to: Monica Crippa (monica.crippa@jrc.ec.europa.eu)

The copyright of individual parts of the supplement might differ from the CC-BY 3.0 licence. 


\section{S1 Comparison of reference 2010 emissions and retrospective scenarios}

Table S1.1 reports the ratios of the STAG_TECH to REF emission scenarios for the global and European scales in 2010. These ratios range from 1.06 to 2.40 for the power and industrial sectors at the global scale, corresponding to a change in technology and implementation of abatement measures; a much higher ratio of 8.6 is indeed obtained for $\mathrm{SO}_{2}$ for road transport, reflecting the use of lower sulfur content fuels for vehicles. Much higher ratios are associated with Europe, due to the strongest impact of the implementation of European legislation. In Europe, for components other than $\mathrm{NH}_{3}$, ratios of 1-3.2 and 6.2-8 are found for the energy sector for gaseous and particulate matter pollutants, respectively; lower ratios ranging from 1.2 to 2.6 are obtained for industry, while higher ratios are found for road transport (ranging from 2.5 to 165 for gaseous components and around 4 for PM). Further discussion on these ratios at sector level is reported in sections 3.2, 3.3 and 3.4 of the manuscript.

Table S1.1: Coupled effect of EU legislation and constant Emission Factor (EF) on pollutant emissions at global and European scales (year 2010). The ratio between STAG_TECH and REF scenarios for the year 2010 is reported for each emission sector. EU27 emission ratios are reported in brackets.

\begin{tabular}{|c|c|c|c|c|c|c|c|c|c|}
\hline \multirow{2}{*}{ Emission sector } & \multicolumn{7}{|c|}{ Emission ratio: STAG_TECH to REF (2010) - Globe (EU27) } \\
\cline { 2 - 10 } & $\mathbf{S O}_{\mathbf{2}}$ & $\mathbf{N O x}$ & $\mathbf{C O}$ & $\mathbf{N M V O C}$ & $\mathbf{N H}_{\mathbf{3}}$ & $\mathbf{P M}_{\mathbf{1 0}}$ & $\mathbf{P M}_{\mathbf{2 . 5}}$ & $\mathbf{B C}$ & $\mathbf{O C}$ \\
\hline \multirow{2}{*}{ Power } & $\begin{array}{c}1.39 \\
(3.19)\end{array}$ & $\begin{array}{c}1.07 \\
(1.70)\end{array}$ & $\begin{array}{c}1.50 \\
(1.00)\end{array}$ & $\begin{array}{c}1.07 \\
(1.10)\end{array}$ & $\begin{array}{c}1.24 \\
(2.72)\end{array}$ & $\begin{array}{c}1.23 \\
(6.28)\end{array}$ & $\begin{array}{c}1.21 \\
(6.49)\end{array}$ & $\begin{array}{c}1.68 \\
(6.18)\end{array}$ & $\begin{array}{c}1.27 \\
(7.99)\end{array}$ \\
\hline \multirow{2}{*}{ Industry } & $\begin{array}{c}1.28 \\
(2.02)\end{array}$ & $\begin{array}{c}1.06 \\
(1.23)\end{array}$ & $\begin{array}{c}2.40 \\
(1.63)\end{array}$ & $\begin{array}{c}1.15 \\
(1.32)\end{array}$ & $\begin{array}{c}1.28 \\
(3.87)\end{array}$ & $\begin{array}{c}1.21 \\
(1.72)\end{array}$ & $\begin{array}{c}1.17 \\
(1.58)\end{array}$ & $\begin{array}{c}1.52 \\
(2.60)\end{array}$ & $\begin{array}{c}1.11 \\
(2.42)\end{array}$ \\
\hline \multirow{2}{*}{ Road Transport } & $\begin{array}{c}8.55 \\
(164.76)\end{array}$ & $\begin{array}{c}1.57 \\
(2.46)\end{array}$ & $\begin{array}{c}1.75 \\
(6.05)\end{array}$ & $\begin{array}{c}1.62 \\
(5.54)\end{array}$ & $\begin{array}{c}0.70 \\
(0.18)\end{array}$ & $\begin{array}{c}2.09 \\
(4.51)\end{array}$ & $\begin{array}{c}2.09 \\
(4.50)\end{array}$ & $\begin{array}{c}1.98 \\
(4.11)\end{array}$ & $\begin{array}{c}2.11 \\
(4.48)\end{array}$ \\
\hline
\end{tabular}

Table S1.2 shows the ratios between STAG_ENERGY and REF global and European emissions in 2010. Global ratios of STAG_ENERGY to REF emissions for energy are 0.12-0.26, for industry between 0.37 and 0.61 , and for road-transport between 0.36 and 0.75 . For Europe these ratios are $0.12-0.35$ for the power generation sector, $1.59-1.93$ for industry and $0.41-0.70$ for road transport. 
Table S1.2: Impact of 1970 energy consumption on 2010 pollutant emissions considering current energy efficiency and fuel mix. The ratio between STAG_ENERGY and REF scenarios for the year 2010 is reported for each emission sector. EU27 emission ratios are reported in brackets.

\begin{tabular}{|c|c|c|c|c|c|c|c|c|c|}
\hline \multirow{2}{*}{ Emission sector } & \multicolumn{7}{|c|}{ Emission ratio: STAG_ENERGY to REF (2010) - Globe (EU27) } \\
\cline { 2 - 10 } & $\mathbf{S O}_{\mathbf{2}}$ & $\mathbf{N O x}$ & $\mathbf{C O}$ & $\mathbf{N M V O C}$ & $\mathbf{N H}_{\mathbf{3}}$ & $\mathbf{P M}_{\mathbf{1 0}}$ & $\mathbf{P M}_{2.5}$ & $\mathbf{B C}$ & $\mathbf{O C}$ \\
\hline \multirow{2}{*}{ Power } & $\begin{array}{c}0.14 \\
(0.13)\end{array}$ & $\begin{array}{c}0.18 \\
(0.27)\end{array}$ & $\begin{array}{c}0.25 \\
(0.31)\end{array}$ & $\begin{array}{c}0.26 \\
(0.35)\end{array}$ & $\begin{array}{c}0.11 \\
(0.17)\end{array}$ & $\begin{array}{c}0.16 \\
(0.15)\end{array}$ & $\begin{array}{c}0.16 \\
(0.16)\end{array}$ & $\begin{array}{c}0.12 \\
(0.12)\end{array}$ & $\begin{array}{c}0.16 \\
(0.17)\end{array}$ \\
\hline Industry & $\begin{array}{c}0.44 \\
(1.81)\end{array}$ & $\begin{array}{c}0.61 \\
(1.83)\end{array}$ & $\begin{array}{c}0.38 \\
(1.93)\end{array}$ & $\begin{array}{c}0.46 \\
(1.84)\end{array}$ & $\begin{array}{c}0.50 \\
(1.59)\end{array}$ & $\begin{array}{c}0.42 \\
(1.75)\end{array}$ & $\begin{array}{c}0.43 \\
(1.76)\end{array}$ & $\begin{array}{c}0.40 \\
(1.78)\end{array}$ & $\begin{array}{c}0.37 \\
(1.70)\end{array}$ \\
\hline Road Transport & $\begin{array}{c}0.75 \\
(0.41)\end{array}$ & $\begin{array}{c}0.59 \\
(0.46)\end{array}$ & $\begin{array}{c}0.62 \\
(0.59)\end{array}$ & $\begin{array}{c}0.64 \\
(0.70)\end{array}$ & $\begin{array}{c}0.36 \\
(0.42)\end{array}$ & $\begin{array}{c}0.64 \\
(0.42)\end{array}$ & $\begin{array}{c}0.64 \\
(0.42)\end{array}$ & $\begin{array}{c}0.61 \\
(0.44)\end{array}$ & $\begin{array}{c}0.63 \\
(0.42)\end{array}$ \\
\hline
\end{tabular}

\section{S2 Scenarios overview: emissions comparison (2010)}

In this section, from Table S2.1 to Table S2.9 2010 pollutant emissions are reported for the STAG_ENERGY, STAG_TECH and REF scenarios, in decreasing order of magnitude of STAG_ENERGY for 24 world regions. All anthropogenic emission sectors are considered with the exception of large scale biomass burning, international shipping and aviation, and the sum of regional emissions corresponds to the world totals. China, India and USA are the countries contributing most to today's gaseous emissions ( $\mathrm{SO}_{2}, \mathrm{NOx}$ and $\mathrm{CO}$ for the REF case), as well as to the STAG_ENERGY scenario, due to their high consumption. In addition to China and India, also Africa contributes significantly to actual global PM emissions, while industrialized countries are contributing less due to the deployment of cleaner technologies and particulate abatement measures. Among all world regions, Europe shows the strongest differences between the STAG_TECH and REF scenarios due to the role played by European legislation and abatement measures included in the STAG_TECH scenario. 
Table S2.1 Comparison of $\mathrm{SO}_{2}$ emission scenarios per world region (year 2010). Emissions are expressed in $\mathrm{Gg} \mathrm{SO}_{2} / \mathrm{yr}$ and include all emission sectors; numbers in brackets refer to the corresponding power, industry and transport emissions.

\begin{tabular}{|c|c|c|c|}
\hline SO2 - 2010 & STAG_ENERGY & STAG_TECH & REF_2010 \\
\hline Globe & $\begin{array}{c}36932 \\
(6651.1 ; 11077.7 ; 582.2) \\
\end{array}$ & $\begin{array}{c}125600 \\
(67726.8 ; 32623.3 ; 6612.9) \\
\end{array}$ & $\begin{array}{c}93700 \\
(48841.5 ; 25464.2 ; 773.2) \\
\end{array}$ \\
\hline China + & $\begin{array}{c}22567 \\
(2105 ; 2935.1 ; 16.7) \\
\end{array}$ & $\begin{array}{c}37800 \\
(13343.7 ; 17538.8 ; 821.3) \\
\end{array}$ & $\begin{array}{c}31600 \\
(11103 ; 14298.5 ; 48.9) \\
\end{array}$ \\
\hline India + & $\begin{array}{c}5004 \\
(290.2 ; 669.7 ; 52)\end{array}$ & $\begin{array}{c}11500 \\
(6167.3 ; 2703 ; 449.8)\end{array}$ & $\begin{array}{c}10900 \\
(6167.3 ; 2505.3 ; 53)\end{array}$ \\
\hline Southern Africa & $\begin{array}{c}2795 \\
(2093.5 ; 261.7 ; 19.8) \\
\end{array}$ & $\begin{array}{c}2800 \\
(1996.2 ; 304.4 ; 128.7) \\
\end{array}$ & $\begin{array}{c}2700 \\
(1996.2 ; 291.3 ; 18.9) \\
\end{array}$ \\
\hline Middle East & $\begin{array}{c}2187 \\
(137.5 ; 192.2 ; 85.5) \\
\end{array}$ & $\begin{array}{c}8300 \\
(5446.5 ; 1334.8 ; 743.1) \\
\end{array}$ & $\begin{array}{c}7500 \\
(5446.5 ; 1223.6 ; 102.3) \\
\end{array}$ \\
\hline OECD Europe & $\begin{array}{c}2104 \\
(60.1 ; 1014.3 ; 1.8)\end{array}$ & $\begin{array}{c}8700 \\
(5165.7 ; 1310.3 ; 828.3)\end{array}$ & $\begin{array}{c}3200 \\
(1153.7 ; 622.1 ; 4.4)\end{array}$ \\
\hline USA & $\begin{array}{c}2041 \\
(445.5 ; 1564.8 ; 6.8)\end{array}$ & $\begin{array}{c}20800 \\
(17059.7 ; 2213.4 ; 818.2)\end{array}$ & $\begin{array}{c}10100 \\
(8493.8 ; 839.6 ; 14.5) \\
\end{array}$ \\
\hline Southeastern Asia & $\begin{array}{c}1761 \\
(190.4 ; 432.8 ; 167.4) \\
\end{array}$ & $\begin{array}{c}2800 \\
(895.8 ; 960.7 ; 331.2)\end{array}$ & $\begin{array}{c}2500 \\
(895.8 ; 804.6 ; 201.2)\end{array}$ \\
\hline Rest South America & $\begin{array}{c}1413 \\
(214.4 ; 185.1 ; 6.2) \\
\end{array}$ & $\begin{array}{c}2500 \\
(939.7 ; 547.1 ; 312.6) \\
\end{array}$ & $\begin{array}{c}2200 \\
(939.7 ; 484.9 ; 19.5) \\
\end{array}$ \\
\hline Russia + & $\begin{array}{c}1309 \\
(214.9 ; 325.7 ; 27.2)\end{array}$ & $\begin{array}{c}4200 \\
(2980.6 ; 317 ; 205.5)\end{array}$ & $\begin{array}{c}2700 \\
(1580.3 ; 281.1 ; 57.4)\end{array}$ \\
\hline Korea & $\begin{array}{c}1276 \\
(93.9 ; 517.6 ; 16.7) \\
\end{array}$ & $\begin{array}{c}1800 \\
(426.3 ; 821.9 ; 165.7) \\
\end{array}$ & $\begin{array}{c}1600 \\
(426.3 ; 727.6 ; 20.4) \\
\end{array}$ \\
\hline Central Europe & $\begin{array}{c}1069 \\
(323.3 ; 476.3 ; 0.6)\end{array}$ & $\begin{array}{c}5500 \\
(4463.3 ; 374.5 ; 142.7)\end{array}$ & $\begin{array}{c}3000 \\
(2257.1 ; 225.7 ; 1.5)\end{array}$ \\
\hline Indonesia + & $\begin{array}{c}1037 \\
(81 ; 25.3 ; 78.3) \\
\end{array}$ & $\begin{array}{c}2200 \\
(1041.6 ; 617.4 ; 145.3) \\
\end{array}$ & $\begin{array}{c}2000 \\
(1041.6 ; 516.2 ; 92.9) \\
\end{array}$ \\
\hline Brazil & $\begin{array}{c}951 \\
(24.8 ; 142.6 ; 3.6) \\
\end{array}$ & $\begin{array}{c}1600 \\
(217.2 ; 595 ; 320.3) \\
\end{array}$ & $\begin{array}{c}1200 \\
(216.7 ; 476.6 ; 12) \\
\end{array}$ \\
\hline Japan & $\begin{array}{c}870 \\
(9.1 ; 422.6 ; 3.1)\end{array}$ & $\begin{array}{c}1700 \\
(292 ; 786.3 ; 117.8)\end{array}$ & $\begin{array}{c}1100 \\
(234.9 ; 380.8 ; 3.5)\end{array}$ \\
\hline Turkey & $\begin{array}{c}772 \\
(0.7 ; 43.3 ; 7.4) \\
\end{array}$ & $\begin{array}{c}1600 \\
(724.1 ; 383.4 ; 111) \\
\end{array}$ & $\begin{array}{c}1500 \\
(724.1 ; 357.6 ; 16) \\
\end{array}$ \\
\hline Asia-Stan & $\begin{array}{c}658 \\
(51.2 ; 756.5 ; 6.9)\end{array}$ & $\begin{array}{c}1900 \\
(1189.4 ; 334 ; 19.7)\end{array}$ & $\begin{array}{c}1800 \\
(1189.4 ; 262.2 ; 7.3)\end{array}$ \\
\hline Rest Central America & $\begin{array}{c}571 \\
(53.5 ; 202 ; 3.1) \\
\end{array}$ & $\begin{array}{c}1400 \\
(781.1 ; 250.5 ; 65.3) \\
\end{array}$ & $\begin{array}{c}1300 \\
(781 ; 226.5 ; 4.1)\end{array}$ \\
\hline Oceania & $\begin{array}{c}502 \\
(12.1 ; 91.2 ; 3.4)\end{array}$ & $\begin{array}{c}1600 \\
(870 ; 192.9 ; 145.5)\end{array}$ & $\begin{array}{c}1100 \\
(602.6 ; 121.3 ; 9.8)\end{array}$ \\
\hline Mexico & $\begin{array}{c}494 \\
(40.4 ; 108.9 ; 21.8) \\
\end{array}$ & $\begin{array}{c}1600 \\
(899.3 ; 239.4 ; 228.3) \\
\end{array}$ & $\begin{array}{c}1300 \\
(845.5 ; 175.6 ; 21.6) \\
\end{array}$ \\
\hline Northern Africa & $\begin{array}{c}488 \\
(12.1 ; 45.7 ; 31)\end{array}$ & $\begin{array}{c}1700 \\
(985.9 ; 206.1 ; 255.1)\end{array}$ & $\begin{array}{c}1500 \\
(985.9 ; 191.3 ; 32.1)\end{array}$ \\
\hline Canada & $\begin{array}{c}474 \\
(18.5 ; 84.4 ; 0)\end{array}$ & $\begin{array}{c}1300 \\
(621.5 ; 199.8 ; 90)\end{array}$ & $\begin{array}{c}1000 \\
(540.9 ; 113 ; 1.6)\end{array}$ \\
\hline Ukraine + & $\begin{array}{c}424 \\
(143.7 ; 523.5 ; 7)\end{array}$ & $\begin{array}{c}1300 \\
(919.1 ; 196.4 ; 41)\end{array}$ & $\begin{array}{c}1200 \\
(918.4 ; 156.2 ; 10.7)\end{array}$ \\
\hline Western Africa & $\begin{array}{c}359 \\
(8.8 ; 37.4 ; 11.1) \\
\end{array}$ & $\begin{array}{c}600 \\
(145 ; 124.9 ; 72.2) \\
\end{array}$ & $\begin{array}{c}500 \\
(145 ; 115.7 ; 12.9) \\
\end{array}$ \\
\hline Eastern Africa & $\begin{array}{c}269 \\
(26.9 ; 18.8 ; 4.9) \\
\end{array}$ & $\begin{array}{c}400 \\
(156 ; 71.3 ; 54.2) \\
\end{array}$ & $\begin{array}{c}400 \\
(156 ; 66.7 ; 6.7) \\
\end{array}$ \\
\hline
\end{tabular}


Table S2.2 Comparison of $\mathrm{NO}_{\mathrm{x}}$ emission scenarios per world region (year 2010). Emissions are expressed in $\mathrm{Gg} \mathrm{NO}_{\mathrm{x}} / \mathrm{yr}$ and include all emission sectors; numbers in brackets refer to the corresponding power, industry and transport emissions.

\begin{tabular}{|c|c|c|c|}
\hline NOx - 2010 & STAG_ENERGY & STAG_TECH & REF_2010 \\
\hline Globe & $\begin{array}{c}53595 \\
(5725.4 ; 10071.8 ; 16151.4)\end{array}$ & $\begin{array}{c}115700 \\
(33491.5 ; 17496.8 ; 43066.9)\end{array}$ & $\begin{array}{c}96900 \\
(31363.4 ; 16502.8 ; 27387.5)\end{array}$ \\
\hline China + & $\begin{array}{c}14806 \\
(1350.6 ; 1437.3 ; 1227.4)\end{array}$ & $\begin{array}{c}27200 \\
(9674.8 ; 6989.7 ; 5303.1)\end{array}$ & $\begin{array}{c}25100 \\
(9674.8 ; 6972.8 ; 3193.1)\end{array}$ \\
\hline USA & $\begin{array}{c}6680 \\
(625.7 ; 2549.9 ; 2320)\end{array}$ & $\begin{array}{c}13400 \\
(3925.4 ; 1368.7 ; 5683.3)\end{array}$ & $\begin{array}{c}12500 \\
(3423.4 ; 1368.2 ; 5337.4)\end{array}$ \\
\hline India + & $\begin{array}{c}5860 \\
(193.4 ; 359.2 ; 2107.5) \\
\end{array}$ & $\begin{array}{c}10300 \\
(4169 ; 1467.6 ; 2417) \\
\end{array}$ & $\begin{array}{c}9900 \\
(4169 ; 1382.9 ; 2148.1) \\
\end{array}$ \\
\hline OECD Europe & $\begin{array}{c}4628 \\
(391 ; 1483.1 ; 1226.7) \\
\end{array}$ & $\begin{array}{c}13000 \\
(2838 ; 1129.8 ; 6953.5) \\
\end{array}$ & $\begin{array}{c}7200 \\
(1514.6 ; 881.4 ; 2674.8)\end{array}$ \\
\hline Middle East & $\begin{array}{c}3688 \\
(106.2 ; 98 ; 2416.9)\end{array}$ & $\begin{array}{c}8200 \\
(2396.9 ; 699 ; 4604.7)\end{array}$ & $\begin{array}{c}6100 \\
(2396.9 ; 679.8 ; 2549.5)\end{array}$ \\
\hline Southeastern Asia & $\begin{array}{c}2602 \\
(157.1 ; 368.4 ; 794.5)\end{array}$ & $\begin{array}{c}4100 \\
(690.1 ; 710.4 ; 1653.3)\end{array}$ & $\begin{array}{c}3300 \\
(690.1 ; 667.8 ; 959.9)\end{array}$ \\
\hline Russia + & $\begin{array}{c}2365 \\
(1006.1 ; 380.5 ; 340.6)\end{array}$ & $\begin{array}{c}4900 \\
(2071.6 ; 331.9 ; 1739.5)\end{array}$ & $\begin{array}{c}3900 \\
(2071.6 ; 324.5 ; 814.4)\end{array}$ \\
\hline Mexico & $\begin{array}{c}1799 \\
(22.1 ; 93.5 ; 1303.8)\end{array}$ & $\begin{array}{c}2200 \\
(323.8 ; 159 ; 1442)\end{array}$ & $\begin{array}{c}2100 \\
(323.8 ; 150.7 ; 1303)\end{array}$ \\
\hline Southern Africa & $\begin{array}{c}1739 \\
(970.3 ; 157.6 ; 284.7)\end{array}$ & $\begin{array}{c}2200 \\
(938.4 ; 244.4 ; 715.7)\end{array}$ & $\begin{array}{c}1800 \\
(938.4 ; 188 ; 372.6)\end{array}$ \\
\hline Brazil & $\begin{array}{c}1730 \\
(5.6 ; 135.2 ; 185.9)\end{array}$ & $\begin{array}{c}3600 \\
(190.7 ; 471.1 ; 1811.6)\end{array}$ & $\begin{array}{c}3100 \\
(190.7 ; 451.8 ; 1310.6)\end{array}$ \\
\hline Indonesia + & $\begin{array}{c}1689 \\
(25.2 ; 21.1 ; 457.9)\end{array}$ & $\begin{array}{c}2500 \\
(498.4 ; 534.7 ; 742)\end{array}$ & $\begin{array}{c}2200 \\
(498.4 ; 475.9 ; 506.8)\end{array}$ \\
\hline Rest South America & $\begin{array}{c}1580 \\
(124.6 ; 163.7 ; 461.5)\end{array}$ & $\begin{array}{c}3100 \\
(532.6 ; 419.8 ; 1515.4)\end{array}$ & $\begin{array}{c}2900 \\
(532.6 ; 414.7 ; 1313.7)\end{array}$ \\
\hline Japan & $\begin{array}{c}1554 \\
(42.9 ; 539.7 ; 329.1) \\
\end{array}$ & $\begin{array}{c}3800 \\
(564.2 ; 670 ; 1881.9)\end{array}$ & $\begin{array}{c}2200 \\
(485.8 ; 486.3 ; 527.4)\end{array}$ \\
\hline Central Europe & $\begin{array}{c}1295 \\
(221.2 ; 562 ; 361)\end{array}$ & $\begin{array}{c}3200 \\
(1085.5 ; 261.8 ; 1374.4)\end{array}$ & $\begin{array}{c}2300 \\
(861.1 ; 257.7 ; 730.2)\end{array}$ \\
\hline Korea & $\begin{array}{c}1194 \\
(42.7 ; 195.2 ; 516.4) \\
\end{array}$ & $\begin{array}{c}1700 \\
(426.4 ; 382.4 ; 663.9) \\
\end{array}$ & $\begin{array}{c}1700 \\
(426.4 ; 381.3 ; 664.4) \\
\end{array}$ \\
\hline Western Africa & $\begin{array}{c}1123 \\
(6 ; 57.7 ; 420.1)\end{array}$ & $\begin{array}{c}1500 \\
(112 ; 345.5 ; 493.1)\end{array}$ & $\begin{array}{c}1300 \\
(112 ; 189.5 ; 487)\end{array}$ \\
\hline Northern Africa & $\begin{array}{c}930 \\
(70 ; 28.5 ; 592.8)\end{array}$ & $\begin{array}{c}1900 \\
(488 ; 142.6 ; 1053.6)\end{array}$ & $\begin{array}{c}1500 \\
(488 ; 129.9 ; 632.9)\end{array}$ \\
\hline Canada & $\begin{array}{c}875 \\
(31.7 ; 194.3 ; 23.6)\end{array}$ & $\begin{array}{c}1700 \\
(307.1 ; 261.3 ; 615.2)\end{array}$ & $\begin{array}{c}1700 \\
(307.1 ; 260.1 ; 572.8)\end{array}$ \\
\hline Oceania & $\begin{array}{c}646 \\
(27.8 ; 109.9 ; 89.3)\end{array}$ & $\begin{array}{c}2100 \\
(582.1 ; 154.6 ; 952.2)\end{array}$ & $\begin{array}{c}1400 \\
(582.1 ; 149.3 ; 289)\end{array}$ \\
\hline Rest Central America & $\begin{array}{c}605 \\
(37.4 ; 93.5 ; 178.6)\end{array}$ & $\begin{array}{c}1100 \\
(485.5 ; 139.3 ; 253.5)\end{array}$ & $\begin{array}{c}1100 \\
(485.5 ; 133.4 ; 225.7)\end{array}$ \\
\hline Asia-Stan & $\begin{array}{c}594 \\
(114.4 ; 493 ; 84.9) \\
\end{array}$ & $\begin{array}{c}1100 \\
(412.3 ; 194.6 ; 206.1) \\
\end{array}$ & $\begin{array}{c}900 \\
(412.3 ; 190.2 ; 88.8) \\
\end{array}$ \\
\hline Ukraine + & $\begin{array}{c}564 \\
(121.9 ; 519 ; 109)\end{array}$ & $\begin{array}{c}1100 \\
(392 ; 177.2 ; 318.5)\end{array}$ & $\begin{array}{c}900 \\
(392 ; 156.5 ; 178.7)\end{array}$ \\
\hline Eastern Africa & $\begin{array}{c}534 \\
(21.9 ; 12.2 ; 160.7)\end{array}$ & $\begin{array}{c}800 \\
(141.1 ; 82.9 ; 223.5)\end{array}$ & $\begin{array}{c}700 \\
(141.1 ; 51.6 ; 207.2)\end{array}$ \\
\hline Turkey & $\begin{array}{c}527 \\
(9.5 ; 19.2 ; 158.6)\end{array}$ & $\begin{array}{c}1100 \\
(245.9 ; 158.4 ; 449.9)\end{array}$ & $\begin{array}{c}900 \\
(245.9 ; 158.4 ; 299.4)\end{array}$ \\
\hline
\end{tabular}


Table S2.3 Comparison of CO emission scenarios per world region (year 2010). Emissions are expressed in $\mathrm{Gg} \mathrm{CO} / \mathrm{yr}$ and include all emission sectors; numbers in brackets refer to the corresponding power, industry and transport emissions.

\begin{tabular}{|c|c|c|c|}
\hline CO - 2010 & STAG_ENERGY & STAG_TECH & REF_2010 \\
\hline Globe & $\begin{array}{c}523999 \\
(1590.4 ; 17591.2 ; 103046.7) \\
\end{array}$ & $\begin{array}{c}811600 \\
(9721.2 ; 111877.5 ; 288233) \\
\end{array}$ & $\begin{array}{c}620000 \\
(6490.8 ; 46679.2 ; 165060) \\
\end{array}$ \\
\hline China + & $\begin{array}{c}161425 \\
(115.2 ; 5578.6 ; 9794.5)\end{array}$ & $\begin{array}{c}218500 \\
(974.5 ; 46438 ; 47068.3)\end{array}$ & $\begin{array}{c}165500 \\
(960.9 ; 27508.1 ; 12994.5)\end{array}$ \\
\hline India + & $\begin{array}{c}95176 \\
(21.8 ; 1938 ; 16828.6)\end{array}$ & $\begin{array}{c}108100 \\
(736.2 ; 13961.8 ; 22461)\end{array}$ & $\begin{array}{c}96200 \\
(729.3 ; 7415.7 ; 17099.9)\end{array}$ \\
\hline Southeastern Asia & $\begin{array}{c}45199 \\
(10.4 ; 749.5 ; 7751.3) \\
\end{array}$ & $\begin{array}{c}59400 \\
(271.4 ; 5094.9 ; 18102)\end{array}$ & $\begin{array}{c}48300 \\
(262.9 ; 1513.5 ; 10600.1)\end{array}$ \\
\hline Western Africa & $\begin{array}{c}37693 \\
(1.5 ; 365.4 ; 4166.8)\end{array}$ & $\begin{array}{c}55600 \\
(38.4 ; 18635.5 ; 4608.9)\end{array}$ & $\begin{array}{c}38200 \\
(37.9 ; 1234.1 ; 4608.9)\end{array}$ \\
\hline Indonesia + & $\begin{array}{c}35906 \\
(1.2 ; 46 ; 13889.5)\end{array}$ & $\begin{array}{c}47000 \\
(101.2 ; 7751 ; 18214.8)\end{array}$ & $\begin{array}{c}36200 \\
(100.3 ; 1032.4 ; 14094.7)\end{array}$ \\
\hline Brazil & $\begin{array}{c}24336 \\
(0.3 ; 219.3 ; 2542.6) \\
\end{array}$ & $\begin{array}{c}30700 \\
(78.8 ; 1121.8 ; 8409.4) \\
\end{array}$ & $\begin{array}{c}27800 \\
(71.4 ; 732.7 ; 5868.9) \\
\end{array}$ \\
\hline Eastern Africa & $\begin{array}{c}20205 \\
(6.9 ; 49.4 ; 713.4) \\
\end{array}$ & $\begin{array}{c}24000 \\
(55.2 ; 3680.5 ; 1011)\end{array}$ & $\begin{array}{c}20500 \\
(54.1 ; 244.6 ; 961.5) \\
\end{array}$ \\
\hline USA & $\begin{array}{c}19620 \\
(263.7 ; 2140.2 ; 8610.5)\end{array}$ & $\begin{array}{c}48200 \\
(1030 ; 1443.6 ; 36120.6)\end{array}$ & $\begin{array}{c}47700 \\
(896.4 ; 1148.4 ; 36028.7)\end{array}$ \\
\hline Southern Africa & $\begin{array}{c}16935 \\
(33.3 ; 591.3 ; 1158.4) \\
\end{array}$ & $\begin{array}{c}23500 \\
(41.5 ; 4787.3 ; 3619)\end{array}$ & $\begin{array}{c}17500 \\
(41.3 ; 711.9 ; 1686.2)\end{array}$ \\
\hline Middle East & $\begin{array}{c}14267 \\
(42.2 ; 31 ; 12254.2) \\
\end{array}$ & $\begin{array}{c}25200 \\
(606.9 ; 495.1 ; 22315.5)\end{array}$ & $\begin{array}{c}15300 \\
(604.7 ; 176.7 ; 12735.9) \\
\end{array}$ \\
\hline Rest South America & $\begin{array}{c}13839 \\
(14 ; 237.1 ; 3507.3) \\
\end{array}$ & $\begin{array}{c}27500 \\
(178.6 ; 905.3 ; 16675.2)\end{array}$ & $\begin{array}{c}25800 \\
(111.9 ; 587.5 ; 15304.4)\end{array}$ \\
\hline OECD Europe & $\begin{array}{c}13492 \\
(153.9 ; 1414.1 ; 2173.4)\end{array}$ & $\begin{array}{c}42600 \\
(3131.6 ; 1381.5 ; 27760.7)\end{array}$ & $\begin{array}{c}15400 \\
(599.8 ; 791.9 ; 3635)\end{array}$ \\
\hline Mexico & $\begin{array}{c}9627 \\
(2.8 ; 62.1 ; 7373.3) \\
\end{array}$ & $\begin{array}{c}9800 \\
(133 ; 224.3 ; 7313.9) \\
\end{array}$ & $\begin{array}{c}9600 \\
(77.8 ; 100.1 ; 7271.2) \\
\end{array}$ \\
\hline Rest Central America & $\begin{array}{c}7120 \\
(10.4 ; 213.5 ; 3434.9)\end{array}$ & $\begin{array}{c}8700 \\
(163.7 ; 462.9 ; 4727.2)\end{array}$ & $\begin{array}{c}8000 \\
(144.7 ; 293.8 ; 4180.1)\end{array}$ \\
\hline Central Europe & $\begin{array}{c}6725 \\
(78.7 ; 688.9 ; 1116.1)\end{array}$ & $\begin{array}{c}11200 \\
(397.9 ; 444.1 ; 5096.5)\end{array}$ & $\begin{array}{c}7700 \\
(172.4 ; 322.3 ; 2004.9)\end{array}$ \\
\hline Russia + & $\begin{array}{c}6131 \\
(630.5 ; 163.3 ; 986.6) \\
\end{array}$ & $\begin{array}{c}14200 \\
(871.3 ; 172.2 ; 8827.7)\end{array}$ & $\begin{array}{c}9000 \\
(861.3 ; 139.5 ; 3631.5)\end{array}$ \\
\hline Japan & $\begin{array}{c}4800 \\
(10.5 ; 323.7 ; 2343.3)\end{array}$ & $\begin{array}{c}21700 \\
(244.2 ; 776.8 ; 18528.5)\end{array}$ & $\begin{array}{c}5400 \\
(124.8 ; 291.7 ; 2800.5)\end{array}$ \\
\hline Korea & $\begin{array}{c}4549 \\
(2.9 ; 986.3 ; 1217.1) \\
\end{array}$ & $\begin{array}{c}5400 \\
(102 ; 1572 ; 1701.3) \\
\end{array}$ & $\begin{array}{c}4900 \\
(95 ; 1253.8 ; 1496.4) \\
\end{array}$ \\
\hline Turkey & $\begin{array}{c}3812 \\
(3.6 ; 40.5 ; 163.3)\end{array}$ & $\begin{array}{c}4800 \\
(64.8 ; 382.8 ; 1075.5)\end{array}$ & $\begin{array}{c}4400 \\
(62.4 ; 334.6 ; 699.5)\end{array}$ \\
\hline Northern Africa & $\begin{array}{c}2700 \\
(40 ; 21 ; 1387.8) \\
\end{array}$ & $\begin{array}{c}6000 \\
(148.2 ; 986.5 ; 3620) \\
\end{array}$ & $\begin{array}{c}3300 \\
(147.8 ; 94.5 ; 1839.2) \\
\end{array}$ \\
\hline Oceania & $\begin{array}{c}2691 \\
(9.4 ; 65.2 ; 387.3)\end{array}$ & $\begin{array}{c}7300 \\
(96.2 ; 217.8 ; 4741.1)\end{array}$ & $\begin{array}{c}3200 \\
(94.4 ; 94.5 ; 811)\end{array}$ \\
\hline Ukraine + & $\begin{array}{c}2613 \\
(56.6 ; 606.8 ; 354.6)\end{array}$ & $\begin{array}{c}3800 \\
(104.8 ; 257.1 ; 1412.7)\end{array}$ & $\begin{array}{c}3000 \\
(104.8 ; 180.8 ; 696.3)\end{array}$ \\
\hline Asia-Stan & $\begin{array}{c}2083 \\
(69 ; 954.8 ; 665.4)\end{array}$ & $\begin{array}{c}3100 \\
(73.6 ; 462.6 ; 1536) \\
\end{array}$ & $\begin{array}{c}2200 \\
(73.6 ; 335.3 ; 748.9)\end{array}$ \\
\hline Canada & $\begin{array}{c}2012 \\
(11.6 ; 105.2 ; 226.6)\end{array}$ & $\begin{array}{c}5200 \\
(77.2 ; 221.9 ; 3286.8)\end{array}$ & $\begin{array}{c}5100 \\
(61.1 ; 140.9 ; 3261.8)\end{array}$ \\
\hline
\end{tabular}


Table S2.4 Comparison of NMVOC emission scenarios per world region (year 2010). Emissions are expressed in Gg NMVOC/yr and include all emission sectors; numbers in brackets refer to the corresponding power, industry and transport emissions.

\begin{tabular}{|c|c|c|c|}
\hline NMVOC - 2010 & STAG_ENERGY & STAG_TECH & REF_2010 \\
\hline Globe & $\begin{array}{c}120828 \\
(184.8 ; 3835.8 ; 16076.8)\end{array}$ & $\begin{array}{c}151700 \\
(752.3 ; 9620.7 ; 40853.8)\end{array}$ & $\begin{array}{c}134800 \\
(704.9 ; 8396.2 ; 25183.2)\end{array}$ \\
\hline China + & $\begin{array}{c}23070 \\
(17 ; 727.6 ; 1259.5) \\
\end{array}$ & $\begin{array}{c}27100 \\
(122.6 ; 4187.9 ; 4739.5)\end{array}$ & $\begin{array}{c}24200 \\
(122.6 ; 3583.6 ; 2473.2)\end{array}$ \\
\hline India + & $\begin{array}{c}14896 \\
(2.1 ; 353.3 ; 1857.2)\end{array}$ & $\begin{array}{c}15500 \\
(70.9 ; 1402.7 ; 2346.4)\end{array}$ & $\begin{array}{c}15000 \\
(70.9 ; 1341.4 ; 1885.1)\end{array}$ \\
\hline Western Africa & $\begin{array}{c}11407 \\
(0.1 ; 102.5 ; 772.8)\end{array}$ & $\begin{array}{c}11500 \\
(3.1 ; 347.5 ; 855)\end{array}$ & $\begin{array}{c}11500 \\
(3.1 ; 346.2 ; 855)\end{array}$ \\
\hline Middle East & $\begin{array}{c}10758 \\
(2.4 ; 17.4 ; 3103.2)\end{array}$ & $\begin{array}{c}13500 \\
(43.9 ; 163.7 ; 5730.2)\end{array}$ & $\begin{array}{c}11100 \\
(43.9 ; 126.8 ; 3405.9)\end{array}$ \\
\hline Southeastern Asia & $\begin{array}{c}9971 \\
(2.1 ; 144.2 ; 888.8)\end{array}$ & $\begin{array}{c}11000 \\
(19.4 ; 361.7 ; 1936.4)\end{array}$ & $\begin{array}{c}10300 \\
(19.4 ; 332.4 ; 1201)\end{array}$ \\
\hline Eastern Africa & $\begin{array}{c}7745 \\
(0.6 ; 14 ; 146.8)\end{array}$ & $\begin{array}{c}7800 \\
(5.1 ; 70.9 ; 207.7)\end{array}$ & $\begin{array}{c}7800 \\
(5.1 ; 69.4 ; 197.3)\end{array}$ \\
\hline Southern Africa & $\begin{array}{c}7669 \\
(8.1 ; 133.2 ; 300.1)\end{array}$ & $\begin{array}{c}8200 \\
(8.4 ; 183 ; 825.8)\end{array}$ & $\begin{array}{c}7800 \\
(8.4 ; 168.4 ; 422.9)\end{array}$ \\
\hline USA & $\begin{array}{c}5406 \\
(22.6 ; 647.5 ; 868.5)\end{array}$ & $\begin{array}{c}8200 \\
(108.2 ; 474.9 ; 3447.5)\end{array}$ & $\begin{array}{c}7900 \\
(78.5 ; 347.4 ; 3298.3)\end{array}$ \\
\hline Brazil & $\begin{array}{c}4795 \\
(0 ; 165.8 ; 491.2)\end{array}$ & $\begin{array}{c}6200 \\
(7 ; 586.6 ; 1802.8)\end{array}$ & $\begin{array}{c}5400 \\
(6.9 ; 553.8 ; 1067.3)\end{array}$ \\
\hline Indonesia + & $\begin{array}{c}4651 \\
(0.1 ; 9.2 ; 1290.4)\end{array}$ & $\begin{array}{c}5100 \\
(7.5 ; 211 ; 1645.8)\end{array}$ & $\begin{array}{c}4700 \\
(7.5 ; 198.6 ; 1334.8)\end{array}$ \\
\hline OECD Europe & $\begin{array}{c}4254 \\
(35.8 ; 461.4 ; 537.4)\end{array}$ & $\begin{array}{c}8700 \\
(133.2 ; 364.6 ; 4820.3)\end{array}$ & $\begin{array}{c}4500 \\
(118.8 ; 271.1 ; 699.8)\end{array}$ \\
\hline Rest South America & $\begin{array}{c}3556 \\
(1.8 ; 81.9 ; 485.6)\end{array}$ & $\begin{array}{c}5500 \\
(12.1 ; 214.7 ; 2328.2)\end{array}$ & $\begin{array}{c}5000 \\
(11.6 ; 199 ; 1897.8)\end{array}$ \\
\hline Russia + & $\begin{array}{c}2813 \\
(60.1 ; 69.9 ; 421.1)\end{array}$ & $\begin{array}{c}5300 \\
(81.8 ; 80.1 ; 2857.3)\end{array}$ & $\begin{array}{c}4100 \\
(81.8 ; 59.6 ; 1693.1)\end{array}$ \\
\hline Mexico & $\begin{array}{c}2112 \\
(0.2 ; 25.7 ; 1327.6)\end{array}$ & $\begin{array}{c}2100 \\
(7.3 ; 45 ; 1351.2)\end{array}$ & $\begin{array}{c}2100 \\
(6.9 ; 41.4 ; 1308.8)\end{array}$ \\
\hline Korea & $\begin{array}{c}2056 \\
(0.2 ; 117.4 ; 179.8)\end{array}$ & $\begin{array}{c}2200 \\
(8.4 ; 217 ; 233.4)\end{array}$ & $\begin{array}{c}2100 \\
(8.4 ; 168.2 ; 221.3)\end{array}$ \\
\hline Northern Africa & $\begin{array}{c}1971 \\
(2.1 ; 9.1 ; 410.1)\end{array}$ & $\begin{array}{c}2400 \\
(10.8 ; 45.3 ; 878.4)\end{array}$ & $\begin{array}{c}2100 \\
(10.8 ; 41.8 ; 495.2)\end{array}$ \\
\hline Rest Central America & $\begin{array}{c}1844 \\
(0.7 ; 55.4 ; 609.1)\end{array}$ & $\begin{array}{c}2100 \\
(14.7 ; 77.4 ; 830)\end{array}$ & $\begin{array}{c}2000 \\
(14.4 ; 73.3 ; 751.6)\end{array}$ \\
\hline Japan & $\begin{array}{c}1453 \\
(3.9 ; 150.2 ; 411.8)\end{array}$ & $\begin{array}{c}2100 \\
(28.6 ; 172.7 ; 941.4)\end{array}$ & $\begin{array}{c}1500 \\
(28.6 ; 135.3 ; 425.7)\end{array}$ \\
\hline Central Europe & $\begin{array}{c}1325 \\
(10.6 ; 163.3 ; 220.5)\end{array}$ & $\begin{array}{c}2100 \\
(19.5 ; 89.9 ; 955)\end{array}$ & $\begin{array}{c}1500 \\
(19.3 ; 74.8 ; 387.2)\end{array}$ \\
\hline Canada & $\begin{array}{c}908 \\
(1.3 ; 55.7 ; 19.5) \\
\end{array}$ & $\begin{array}{c}1300 \\
(8.6 ; 93.9 ; 317.9)\end{array}$ & $\begin{array}{c}1200 \\
(7 ; 74.6 ; 305.1)\end{array}$ \\
\hline Asia-Stan & $\begin{array}{c}864 \\
(6.4 ; 180.1 ; 237.2)\end{array}$ & $\begin{array}{c}1000 \\
(7.2 ; 73.6 ; 423.8)\end{array}$ & $\begin{array}{c}900 \\
(7.2 ; 64.7 ; 272.3)\end{array}$ \\
\hline Oceania & $\begin{array}{c}713 \\
(1 ; 25.8 ; 66.1)\end{array}$ & $\begin{array}{c}1300 \\
(8.5 ; 47.6 ; 696)\end{array}$ & $\begin{array}{c}800 \\
(8.3 ; 35.6 ; 145.8)\end{array}$ \\
\hline Ukraine + & $\begin{array}{c}552 \\
(5.3 ; 119.2 ; 125.8)\end{array}$ & $\begin{array}{c}800 \\
(10.2 ; 40.2 ; 433.4)\end{array}$ & $\begin{array}{c}700 \\
(10.2 ; 37.4 ; 274.7)\end{array}$ \\
\hline Turkey & $\begin{array}{c}479 \\
(0.3 ; 6.2 ; 46.7)\end{array}$ & $\begin{array}{c}700 \\
(5.3 ; 68.9 ; 250.6)\end{array}$ & $\begin{array}{c}600 \\
(5.3 ; 51.6 ; 163.9)\end{array}$ \\
\hline
\end{tabular}


Table S2.5 Comparison of $\mathrm{NH}_{3}$ emission scenarios per world region (year 2010). Emissions are expressed in $\mathrm{Gg} \mathrm{NH}_{3} / \mathrm{yr}$ and include all emission sectors; numbers in brackets refer to the corresponding power, industry and transport emissions.

\begin{tabular}{|c|c|c|c|}
\hline NH3 - 2010 & STAG_ENERGY & STAG_TECH & REF_2010 \\
\hline Globe & $\begin{array}{c}54099 \\
(9.4 ; 307.2 ; 179.5) \\
\end{array}$ & $\begin{array}{c}54800 \\
(102 ; 791.2 ; 347.5) \\
\end{array}$ & $\begin{array}{c}54800 \\
(82.2 ; 617.2 ; 497.9) \\
\end{array}$ \\
\hline China + & $\begin{array}{c}14078 \\
(0.1 ; 1.4 ; 8.4)\end{array}$ & $\begin{array}{c}14100 \\
(2.8 ; 6.2 ; 6) \\
\end{array}$ & $\begin{array}{c}14100 \\
(2.8 ; 6.2 ; 25.4)\end{array}$ \\
\hline India + & $\begin{array}{c}8595 \\
(0.1 ; 38.1 ; 8.5) \\
\end{array}$ & $\begin{array}{c}8600 \\
(4.9 ; 145.7 ; 6.1)\end{array}$ & $\begin{array}{c}8600 \\
(4.9 ; 145.7 ; 8.6)\end{array}$ \\
\hline OECD Europe & $\begin{array}{c}4166 \\
(0.8 ; 27 ; 19.2) \\
\end{array}$ & $\begin{array}{c}4300 \\
(27.5 ; 80.6 ; 7.5)\end{array}$ & $\begin{array}{c}4200 \\
(7.7 ; 19 ; 46.7) \\
\end{array}$ \\
\hline USA & $\begin{array}{c}3696 \\
(4.9 ; 92.2 ; 62.4)\end{array}$ & $\begin{array}{c}4000 \\
(12.7 ; 122.3 ; 256.4)\end{array}$ & $\begin{array}{c}3900 \\
(12.7 ; 49.5 ; 256.4)\end{array}$ \\
\hline Brazil & $\begin{array}{c}2887 \\
(0 ; 37 ; 1.7)\end{array}$ & $\begin{array}{c}2900 \\
(4.2 ; 123.5 ; 2.1)\end{array}$ & $\begin{array}{c}2900 \\
(4.2 ; 123.5 ; 8.1)\end{array}$ \\
\hline Southeastern Asia & $\begin{array}{c}2794 \\
(0 ; 7.7 ; 7.1)\end{array}$ & $\begin{array}{c}2800 \\
(2.8 ; 39 ; 2.8)\end{array}$ & $\begin{array}{c}2800 \\
(2.8 ; 39 ; 10.3)\end{array}$ \\
\hline Rest South America & $\begin{array}{c}2188 \\
(0.2 ; 12.4 ; 1.5) \\
\end{array}$ & $\begin{array}{c}2200 \\
(3.4 ; 30.7 ; 3.6) \\
\end{array}$ & $\begin{array}{c}2200 \\
(3.4 ; 30.7 ; 8) \\
\end{array}$ \\
\hline Indonesia + & $\begin{array}{c}1899 \\
(0 ; 1.3 ; 3.4) \\
\end{array}$ & $\begin{array}{c}1900 \\
(1.4 ; 29.8 ; 0.7)\end{array}$ & $\begin{array}{c}1900 \\
(1.4 ; 29.8 ; 3.7)\end{array}$ \\
\hline Western Africa & $\begin{array}{c}1798 \\
(0 ; 22.3 ; 0.2)\end{array}$ & $\begin{array}{c}1800 \\
(0.5 ; 75.5 ; 0.3) \\
\end{array}$ & $\begin{array}{c}1800 \\
(0.5 ; 75.5 ; 0.3) \\
\end{array}$ \\
\hline Eastern Africa & $\begin{array}{c}1699 \\
(0.1 ; 3 ; 0.1) \\
\end{array}$ & $\begin{array}{c}1700 \\
(0.8 ; 15 ; 0.1)\end{array}$ & $\begin{array}{c}1700 \\
(0.8 ; 15 ; 0.2)\end{array}$ \\
\hline Central Europe & $\begin{array}{c}1393 \\
(1.1 ; 10.9 ; 2.8) \\
\end{array}$ & $\begin{array}{c}1400 \\
(3.8 ; 11.9 ; 1.9) \\
\end{array}$ & $\begin{array}{c}1400 \\
(3.8 ; 5.1 ; 6.8) \\
\end{array}$ \\
\hline Russia + & $\begin{array}{c}1087 \\
(0.7 ; 1.3 ; 2.4) \\
\end{array}$ & $\begin{array}{c}1100 \\
(3.1 ; 1.5 ; 1.2) \\
\end{array}$ & $\begin{array}{c}1100 \\
(3.1 ; 1.2 ; 12.9) \\
\end{array}$ \\
\hline Southern Africa & $\begin{array}{c}997 \\
(0 ; 18.1 ; 3.1)\end{array}$ & $\begin{array}{c}1000 \\
(0.2 ; 25.2 ; 0.3)\end{array}$ & $\begin{array}{c}1000 \\
(0.2 ; 25.2 ; 4.4)\end{array}$ \\
\hline Middle East & $\begin{array}{c}980 \\
(0.3 ; 1.3 ; 12.9)\end{array}$ & $\begin{array}{c}1000 \\
(13.8 ; 7.8 ; 5.6)\end{array}$ & $\begin{array}{c}1000 \\
(13.8 ; 7.9 ; 19.3)\end{array}$ \\
\hline Mexico & $\begin{array}{c}898 \\
(0 ; 1.6 ; 15.3)\end{array}$ & $\begin{array}{c}900 \\
(2.4 ; 5.2 ; 15.3) \\
\end{array}$ & $\begin{array}{c}900 \\
(2.4 ; 2.5 ; 15.3) \\
\end{array}$ \\
\hline Oceania & $\begin{array}{c}892 \\
(0.1 ; 3.4 ; 6.4)\end{array}$ & $\begin{array}{c}900 \\
(1.6 ; 11.4 ; 1.1)\end{array}$ & $\begin{array}{c}900 \\
(1.6 ; 4.8 ; 12.7)\end{array}$ \\
\hline Turkey & $\begin{array}{c}799 \\
(0 ; 0 ; 0.2)\end{array}$ & $\begin{array}{c}800 \\
(0.2 ; 0.2 ; 1.1)\end{array}$ & $\begin{array}{c}800 \\
(0.2 ; 0.2 ; 1.4)\end{array}$ \\
\hline Northern Africa & $\begin{array}{c}797 \\
(0 ; 1.1 ; 4.1)\end{array}$ & $\begin{array}{c}800 \\
(2.4 ; 4.8 ; 0.8)\end{array}$ & $\begin{array}{c}800 \\
(2.4 ; 4.8 ; 4.2)\end{array}$ \\
\hline Canada & $\begin{array}{c}677 \\
(0.3 ; 8.2 ; 1.3)\end{array}$ & $\begin{array}{c}700 \\
(1.7 ; 27.6 ; 22.8)\end{array}$ & $\begin{array}{c}700 \\
(1.7 ; 11 ; 22.8)\end{array}$ \\
\hline Rest Central America & $\begin{array}{c}596 \\
(0.2 ; 9.3 ; 1.3)\end{array}$ & $\begin{array}{c}600 \\
(3.9 ; 10.9 ; 1.6)\end{array}$ & $\begin{array}{c}600 \\
(3.9 ; 10.9 ; 2)\end{array}$ \\
\hline Ukraine + & $\begin{array}{c}498 \\
(0.2 ; 1.2 ; 0.9)\end{array}$ & $\begin{array}{c}500 \\
(1.1 ; 0.7 ; 0.2)\end{array}$ & $\begin{array}{c}500 \\
(1.1 ; 0.5 ; 2)\end{array}$ \\
\hline Asia-Stan & $\begin{array}{c}498 \\
(0 ; 0.9 ; 1.8)\end{array}$ & $\begin{array}{c}500 \\
(0.2 ; 0.3 ; 0.2)\end{array}$ & $\begin{array}{c}500 \\
(0.2 ; 0.3 ; 2)\end{array}$ \\
\hline Japan & $\begin{array}{c}385 \\
(0.1 ; 7.1 ; 9.3)\end{array}$ & $\begin{array}{c}400 \\
(5.3 ; 12.7 ; 2.6)\end{array}$ & $\begin{array}{c}400 \\
(5.3 ; 6.4 ; 17)\end{array}$ \\
\hline Korea & $\begin{array}{c}297 \\
(0 ; 0.5 ; 5.2)\end{array}$ & $\begin{array}{c}300 \\
(1.2 ; 2.9 ; 7.4)\end{array}$ & $\begin{array}{c}300 \\
(1.2 ; 2.5 ; 7.4)\end{array}$ \\
\hline
\end{tabular}


Table S2.6 Comparison of $\mathbf{P M}_{10}$ emission scenarios per world region (year 2010). Emissions are expressed in $\mathbf{G g} \mathbf{P M}_{10} / \mathbf{y r}$ and include all emission sectors; numbers in brackets refer to the corresponding power, industry and transport emissions.

\begin{tabular}{|c|c|c|c|}
\hline PM10 - 2010 & STAG_ENERGY & STAG_TECH & REF_2010 \\
\hline Globe & $\begin{array}{c}54576 \\
(766.4 ; 3421.8 ; 555.5) \\
\end{array}$ & $\begin{array}{c}67400 \\
(6003.1 ; 9786.7 ; 1831) \\
\end{array}$ & $\begin{array}{c}63700 \\
(4876.7 ; 8117 ; 874) \\
\end{array}$ \\
\hline China + & $\begin{array}{c}17788 \\
(327 ; 883.5 ; 35.6)\end{array}$ & $\begin{array}{c}19960 \\
(1877.3 ; 4731.9 ; 279)\end{array}$ & $\begin{array}{c}19370 \\
(1824.5 ; 4353.4 ; 114.9)\end{array}$ \\
\hline India + & $\begin{array}{c}10383 \\
(82.6 ; 275.6 ; 103.2)\end{array}$ & $\begin{array}{c}12120 \\
(1610.4 ; 1219 ; 134.8)\end{array}$ & $\begin{array}{c}11720 \\
(1413.9 ; 1043 ; 105.6)\end{array}$ \\
\hline Western Africa & $\begin{array}{c}5190 \\
(2.3 ; 61.4 ; 9.6)\end{array}$ & $\begin{array}{c}5330 \\
(5 ; 339.4 ; 12.2)\end{array}$ & $\begin{array}{c}5200 \\
(4.8 ; 206.7 ; 12.2)\end{array}$ \\
\hline Southeastern Asia & $\begin{array}{c}4779 \\
(20.4 ; 90.3 ; 53.7) \\
\end{array}$ & $\begin{array}{c}5030 \\
(101.2 ; 345.3 ; 119.1) \\
\end{array}$ & $\begin{array}{c}4860 \\
(89.4 ; 238.3 ; 65.5) \\
\end{array}$ \\
\hline Brazil & $\begin{array}{c}3159 \\
(0.4 ; 162 ; 8.6) \\
\end{array}$ & $\begin{array}{c}3310 \\
(29.2 ; 595.1 ; 74.7) \\
\end{array}$ & $\begin{array}{c}3250 \\
(28.9 ; 541.2 ; 60.3) \\
\end{array}$ \\
\hline Indonesia + & $\begin{array}{c}3009 \\
(5.9 ; 6.6 ; 50.9) \\
\end{array}$ & $\begin{array}{c}3150 \\
(64.6 ; 200.7 ; 77.2) \\
\end{array}$ & $\begin{array}{c}3060 \\
(55.6 ; 143.8 ; 56.3) \\
\end{array}$ \\
\hline Eastern Africa & $\begin{array}{c}2952 \\
(3.4 ; 8.6 ; 7.9) \\
\end{array}$ & $\begin{array}{c}2990 \\
(8.1 ; 69 ; 11.9) \\
\end{array}$ & $\begin{array}{c}2960 \\
(7.8 ; 42.1 ; 11.6) \\
\end{array}$ \\
\hline Southern Africa & $\begin{array}{c}2516 \\
(145.3 ; 107.9 ; 26.1)\end{array}$ & $\begin{array}{c}2570 \\
(151.4 ; 169.3 ; 25.5)\end{array}$ & $\begin{array}{c}2500 \\
(133.9 ; 128.7 ; 16.4)\end{array}$ \\
\hline USA & $\begin{array}{c}1720 \\
(22.7 ; 686.6 ; 31.1)\end{array}$ & $\begin{array}{c}2320 \\
(452.1 ; 488.1 ; 80.2)\end{array}$ & $\begin{array}{c}2160 \\
(428.4 ; 368.4 ; 60.2)\end{array}$ \\
\hline OECD Europe & $\begin{array}{c}1306 \\
(7.4 ; 344.5 ; 47.9)\end{array}$ & $\begin{array}{c}2430 \\
(466.8 ; 397.3 ; 535.3)\end{array}$ & $\begin{array}{c}1450 \\
(88 ; 220.7 ; 111.1)\end{array}$ \\
\hline Rest South America & $\begin{array}{c}1302 \\
(60.8 ; 59.4 ; 27.1) \\
\end{array}$ & $\begin{array}{c}1400 \\
(98.2 ; 158.3 ; 55.7)\end{array}$ & $\begin{array}{c}1360 \\
(89.3 ; 139.7 ; 45.4)\end{array}$ \\
\hline Central Europe & $\begin{array}{c}913 \\
(14.4 ; 178.8 ; 10.3)\end{array}$ & $\begin{array}{c}1510 \\
(498.8 ; 121.6 ; 82.7)\end{array}$ & $\begin{array}{c}1050 \\
(133.6 ; 83.9 ; 28.8)\end{array}$ \\
\hline Rest Central America & $\begin{array}{c}578 \\
(1.9 ; 40.3 ; 5)\end{array}$ & $\begin{array}{c}620 \\
(33.1 ; 61.4 ; 7.6)\end{array}$ & $\begin{array}{c}610 \\
(32.3 ; 48 ; 6.4)\end{array}$ \\
\hline Russia + & $\begin{array}{c}502 \\
(24.5 ; 33.5 ; 10.8)\end{array}$ & $\begin{array}{c}640 \\
(138.9 ; 35.1 ; 26.7)\end{array}$ & $\begin{array}{c}610 \\
(128.8 ; 28.8 ; 14.9)\end{array}$ \\
\hline Turkey & $\begin{array}{c}469 \\
(0 ; 6.4 ; 4.5)\end{array}$ & $\begin{array}{c}520 \\
(29.4 ; 61.2 ; 18.3)\end{array}$ & $\begin{array}{c}500 \\
(26.8 ; 52.7 ; 9.9)\end{array}$ \\
\hline Mexico & $\begin{array}{c}410 \\
(1.8 ; 22.5 ; 14.7)\end{array}$ & $\begin{array}{c}440 \\
(22.8 ; 43.8 ; 14.6)\end{array}$ & $\begin{array}{c}430 \\
(21.7 ; 36.3 ; 14.5)\end{array}$ \\
\hline Korea & $\begin{array}{c}389 \\
(29.9 ; 96.8 ; 9.8) \\
\end{array}$ & $\begin{array}{c}530 \\
(141.2 ; 182.1 ; 13.1)\end{array}$ & $\begin{array}{c}480 \\
(123.7 ; 149.4 ; 12.9)\end{array}$ \\
\hline Middle East & $\begin{array}{c}339 \\
(3.1 ; 8.5 ; 48.7) \\
\end{array}$ & $\begin{array}{c}460 \\
(76.6 ; 63.5 ; 82.9) \\
\end{array}$ & $\begin{array}{c}410 \\
(73.4 ; 45.3 ; 53.2) \\
\end{array}$ \\
\hline Canada & $\begin{array}{c}303 \\
(2.7 ; 57.9 ; 0.3)\end{array}$ & $\begin{array}{c}430 \\
(69.8 ; 128 ; 8.3)\end{array}$ & $\begin{array}{c}370 \\
(61.2 ; 77.5 ; 8.2)\end{array}$ \\
\hline Oceania & $\begin{array}{c}281 \\
(1.1 ; 24.1 ; 2.8) \\
\end{array}$ & $\begin{array}{c}370 \\
(36.3 ; 54 ; 36.3) \\
\end{array}$ & $\begin{array}{c}320 \\
(33.8 ; 32.5 ; 9) \\
\end{array}$ \\
\hline Northern Africa & $\begin{array}{c}261 \\
(1.2 ; 4.9 ; 31.6)\end{array}$ & $\begin{array}{c}310 \\
(18.6 ; 28.4 ; 54.8)\end{array}$ & $\begin{array}{c}280 \\
(17.6 ; 19.4 ; 31.8)\end{array}$ \\
\hline Ukraine + & $\begin{array}{c}234 \\
(5 ; 84.1 ; 2.1)\end{array}$ & $\begin{array}{c}280 \\
(32.9 ; 38.3 ; 5.9)\end{array}$ & $\begin{array}{c}260 \\
(30.1 ; 25.9 ; 4.1)\end{array}$ \\
\hline Japan & $\begin{array}{c}230 \\
(0.9 ; 54.4 ; 10.7) \\
\end{array}$ & $\begin{array}{c}470 \\
(28 ; 200.5 ; 70.1) \\
\end{array}$ & $\begin{array}{c}260 \\
(18.3 ; 49 ; 18.3) \\
\end{array}$ \\
\hline Asia-Stan & $\begin{array}{c}205 \\
(1.5 ; 123.3 ; 2.6) \\
\end{array}$ & $\begin{array}{c}240 \\
(12.2 ; 55.6 ; 3.8) \\
\end{array}$ & $\begin{array}{c}220 \\
(11.2 ; 42.2 ; 2.7) \\
\end{array}$ \\
\hline
\end{tabular}


Table S2.7 Comparison of $\mathbf{P M}_{2.5}$ emission scenarios per world region (year 2010). Emissions are expressed in $\mathbf{G g} \mathbf{P M}_{2.5} / \mathbf{y r}$ and include all emission sectors; numbers in brackets refer to the corresponding power, industry and transport emissions.

\begin{tabular}{|c|c|c|c|}
\hline PM2.5 - 2010 & STAG_ENERGY & STAG_TECH & REF_2010 \\
\hline Globe & $\begin{array}{c}34594 \\
(484 ; 2943.5 ; 555.5)\end{array}$ & $\begin{array}{c}44100 \\
(3654.1 ; 8096 ; 1827.9)\end{array}$ & $\begin{array}{c}41400 \\
(3011.5 ; 6903.7 ; 874)\end{array}$ \\
\hline China + & $\begin{array}{c}12315 \\
(187.6 ; 770.6 ; 35.6) \\
\end{array}$ & $\begin{array}{c}13660 \\
(1005 ; 4078.9 ; 279) \\
\end{array}$ & $\begin{array}{c}13190 \\
(978.7 ; 3799.8 ; 114.9) \\
\end{array}$ \\
\hline India + & $\begin{array}{c}6974 \\
(63 ; 243.6 ; 103.2)\end{array}$ & $\begin{array}{c}8210 \\
(1176.9 ; 1006.8 ; 134.8)\end{array}$ & $\begin{array}{c}7980 \\
(1063.6 ; 921.9 ; 105.6)\end{array}$ \\
\hline Southeastern Asia & $\begin{array}{c}3356 \\
(9.9 ; 83.7 ; 53.7)\end{array}$ & $\begin{array}{c}3520 \\
(57.4 ; 269.7 ; 119.1)\end{array}$ & $\begin{array}{c}3410 \\
(52.2 ; 215 ; 65.5)\end{array}$ \\
\hline Western Africa & $\begin{array}{c}2611 \\
(1.7 ; 57.2 ; 9.6)\end{array}$ & $\begin{array}{c}2670 \\
(3.4 ; 241.8 ; 12.2)\end{array}$ & $\begin{array}{c}2620 \\
(3.2 ; 194 ; 12.2)\end{array}$ \\
\hline Brazil & $\begin{array}{c}2308 \\
(0.2 ; 89.2 ; 8.6)\end{array}$ & $\begin{array}{c}2580 \\
(13.5 ; 479 ; 74.7)\end{array}$ & $\begin{array}{c}2380 \\
(13.3 ; 298 ; 60.3)\end{array}$ \\
\hline Indonesia + & $\begin{array}{c}2106 \\
(3.8 ; 6 ; 50.9)\end{array}$ & $\begin{array}{c}2190 \\
(39.8 ; 155.7 ; 77.2) \\
\end{array}$ & $\begin{array}{c}2140 \\
(36.1 ; 130.9 ; 56.3) \\
\end{array}$ \\
\hline Eastern Africa & $\begin{array}{c}1414 \\
(2.5 ; 8 ; 7.9) \\
\end{array}$ & $\begin{array}{c}1430 \\
(5 ; 49.4 ; 11.9) \\
\end{array}$ & $\begin{array}{c}1420 \\
(4.7 ; 39.7 ; 11.6) \\
\end{array}$ \\
\hline Southern Africa & $\begin{array}{c}1243 \\
(92.4 ; 82.5 ; 26.1) \\
\end{array}$ & $\begin{array}{c}1280 \\
(93.3 ; 128.6 ; 25.5) \\
\end{array}$ & $\begin{array}{c}1230 \\
(84.3 ; 102 ; 16.4) \\
\end{array}$ \\
\hline (2) & $\begin{array}{c}1050 \\
(14.6 ; 635.8 ; 31.1)\end{array}$ & $\begin{array}{c}1410 \\
(269.9 ; 390.5 ; 79.8)\end{array}$ & $\begin{array}{c}1330 \\
(260.8 ; 341.1 ; 60.2)\end{array}$ \\
\hline Rest South America & $\begin{array}{c}907 \\
(46 ; 36.3 ; 27.1) \\
\end{array}$ & $\begin{array}{c}1010 \\
(69.1 ; 125.9 ; 55.7) \\
\end{array}$ & $\begin{array}{c}950 \\
(63.5 ; 86 ; 45.4) \\
\end{array}$ \\
\hline OECD Europe & $\begin{array}{c}830 \\
(5.1 ; 299 ; 47.9)\end{array}$ & $\begin{array}{c}1690 \\
(262.9 ; 312.6 ; 534.5)\end{array}$ & $\begin{array}{c}940 \\
(51.8 ; 191.7 ; 111.1)\end{array}$ \\
\hline Central Europe & $\begin{array}{c}548 \\
(8.7 ; 155.8 ; 10.3)\end{array}$ & $\begin{array}{c}950 \\
(306.6 ; 97.8 ; 82.5)\end{array}$ & $\begin{array}{c}630 \\
(73.1 ; 71.3 ; 28.8)\end{array}$ \\
\hline Turkey & $\begin{array}{c}355 \\
(0 ; 5.3 ; 4.5)\end{array}$ & $\begin{array}{c}390 \\
(11.7 ; 49.2 ; 18.2) \\
\end{array}$ & $\begin{array}{c}370 \\
(10.6 ; 43.6 ; 9.9) \\
\end{array}$ \\
\hline Russia + & $\begin{array}{c}331 \\
(14.6 ; 28.1 ; 10.8)\end{array}$ & $\begin{array}{c}410 \\
(74.1 ; 28.1 ; 26.7)\end{array}$ & $\begin{array}{c}390 \\
(70 ; 24.2 ; 14.9)\end{array}$ \\
\hline Rest Central America & $\begin{array}{c}312 \\
(1.3 ; 28 ; 5)\end{array}$ & $\begin{array}{c}340 \\
(18.5 ; 48.2 ; 7.6)\end{array}$ & $\begin{array}{c}330 \\
(18 ; 34.4 ; 6.4) \\
\end{array}$ \\
\hline Korea & $\begin{array}{c}272 \\
(22.7 ; 78.6 ; 9.8)\end{array}$ & $\begin{array}{c}350 \\
(82.2 ; 145.6 ; 13.1)\end{array}$ & $\begin{array}{c}320 \\
(73.8 ; 124.8 ; 12.9)\end{array}$ \\
\hline Mexico & $\begin{array}{c}260 \\
(0.7 ; 15.8 ; 14.7)\end{array}$ & $\begin{array}{c}280 \\
(11.2 ; 34.9 ; 14.6)\end{array}$ & $\begin{array}{c}270 \\
(10.9 ; 25.5 ; 14.5)\end{array}$ \\
\hline Middle East & $\begin{array}{c}231 \\
(2.2 ; 7.4 ; 48.7)\end{array}$ & $\begin{array}{c}320 \\
(51.7 ; 50.4 ; 82.8)\end{array}$ & $\begin{array}{c}280 \\
(49.9 ; 39.6 ; 53.2)\end{array}$ \\
\hline Oceania & $\begin{array}{c}223 \\
(0.4 ; 20.4 ; 2.8)\end{array}$ & $\begin{array}{c}290 \\
(12.3 ; 43.2 ; 36.3)\end{array}$ & $\begin{array}{c}240 \\
(11.6 ; 27.6 ; 9)\end{array}$ \\
\hline Canada & $\begin{array}{c}215 \\
(1.4 ; 55 ; 0.3)\end{array}$ & $\begin{array}{c}290 \\
(42.1 ; 102.4 ; 8.3)\end{array}$ & $\begin{array}{c}260 \\
(38.2 ; 73.6 ; 8.2)\end{array}$ \\
\hline Asia-Stan & $\begin{array}{c}161 \\
(1 ; 109.7 ; 2.6)\end{array}$ & $\begin{array}{c}180 \\
(5 ; 45 ; 3.8)\end{array}$ & $\begin{array}{c}170 \\
(4.7 ; 37.6 ; 2.7)\end{array}$ \\
\hline Northern Africa & $\begin{array}{c}156 \\
(0.9 ; 4.3 ; 31.6)\end{array}$ & $\begin{array}{c}190 \\
(12.6 ; 21.2 ; 54.8)\end{array}$ & $\begin{array}{c}170 \\
(12 ; 17.7 ; 31.8)\end{array}$ \\
\hline Ukraine + & $\begin{array}{c}156 \\
(2.8 ; 80 ; 2.1)\end{array}$ & $\begin{array}{c}180 \\
(16.7 ; 30.5 ; 5.9)\end{array}$ & $\begin{array}{c}170 \\
(15.6 ; 24.7 ; 4.1)\end{array}$ \\
\hline Japan & $\begin{array}{c}138 \\
(0.6 ; 43.1 ; 10.7) \\
\end{array}$ & $\begin{array}{c}330 \\
(13.3 ; 160.4 ; 68.6) \\
\end{array}$ & $\begin{array}{c}160 \\
(10.6 ; 38.8 ; 18.3) \\
\end{array}$ \\
\hline
\end{tabular}


Table S2.8 Comparison of BC emission scenarios per world region (year 2010). Emissions are expressed in $\mathrm{Gg} \mathrm{BC/yr}$ and include all emission sectors; numbers in brackets refer to the corresponding power, industry and transport emissions.

\begin{tabular}{|c|c|c|c|}
\hline BC - 2010 & STAG_ENERGY & STAG_TECH & REF_2010 \\
\hline Globe & $\begin{array}{c}3874 \\
(14.6 ; 312.3 ; 395.6)\end{array}$ & $\begin{array}{c}5900 \\
(199.2 ; 1184.8 ; 1287)\end{array}$ & $\begin{array}{c}4700 \\
(118.6 ; 781.7 ; 648.5)\end{array}$ \\
\hline China + & $\begin{array}{c}1511 \\
(5 ; 59.1 ; 19.8)\end{array}$ & $\begin{array}{c}1730 \\
(29.8 ; 327.4 ; 169.3)\end{array}$ & $\begin{array}{c}1590 \\
(25.4 ; 290.5 ; 77.5)\end{array}$ \\
\hline India + & $\begin{array}{c}860 \\
(2.1 ; 52.7 ; 71)\end{array}$ & $\begin{array}{c}1020 \\
(77.8 ; 262.8 ; 92.3)\end{array}$ & $\begin{array}{c}900 \\
(40.7 ; 199.4 ; 72.7)\end{array}$ \\
\hline Western Africa & $\begin{array}{c}357 \\
(0 ; 15.8 ; 6.4)\end{array}$ & $\begin{array}{c}380 \\
(0.4 ; 67.7 ; 8.3)\end{array}$ & $\begin{array}{c}360 \\
(0.3 ; 53.7 ; 8.3)\end{array}$ \\
\hline Southeastern Asia & $\begin{array}{c}259 \\
(0.3 ; 9.5 ; 31.2) \\
\end{array}$ & $\begin{array}{c}330 \\
(3.7 ; 47.3 ; 73.7) \\
\end{array}$ & $\begin{array}{c}270 \\
(3.2 ; 28.5 ; 39.3) \\
\end{array}$ \\
\hline Eastern Africa & $\begin{array}{c}187 \\
(0.1 ; 2.2 ; 5.8)\end{array}$ & $\begin{array}{c}190 \\
(0.5 ; 14.1 ; 8.7)\end{array}$ & $\begin{array}{c}190 \\
(0.4 ; 11.2 ; 8.4)\end{array}$ \\
\hline Indonesia + & $\begin{array}{c}173 \\
(0.3 ; 1.2 ; 20.7) \\
\end{array}$ & $\begin{array}{c}200 \\
(3.2 ; 31.8 ; 36.4) \\
\end{array}$ & $\begin{array}{c}180 \\
(3.1 ; 25.9 ; 25) \\
\end{array}$ \\
\hline Southern Africa & $\begin{array}{c}167 \\
(2.5 ; 12 ; 19.8)\end{array}$ & $\begin{array}{c}180 \\
(6.2 ; 27.2 ; 18.9)\end{array}$ & $\begin{array}{c}160 \\
(2.3 ; 17.2 ; 12)\end{array}$ \\
\hline Brazil & $\begin{array}{c}139 \\
(0 ; 8.1 ; 4.7) \\
\end{array}$ & $\begin{array}{c}280 \\
(0.9 ; 118.7 ; 53.2) \\
\end{array}$ & $\begin{array}{c}180 \\
(0.8 ; 27.1 ; 43.7) \\
\end{array}$ \\
\hline USA & $\begin{array}{c}122 \\
(0.6 ; 61.9 ; 19.6)\end{array}$ & $\begin{array}{c}210 \\
(17.3 ; 76.2 ; 46.5)\end{array}$ & $\begin{array}{c}160 \\
(12.5 ; 33.2 ; 44.9) \\
\end{array}$ \\
\hline OECD Europe & $\begin{array}{c}116 \\
(0.2 ; 22.4 ; 39.8)\end{array}$ & $\begin{array}{c}530 \\
(15.6 ; 37.6 ; 415.1)\end{array}$ & $\begin{array}{c}170 \\
(3 ; 14.1 ; 91)\end{array}$ \\
\hline Rest South America & $\begin{array}{c}83 \\
(1.2 ; 5 ; 21.1) \\
\end{array}$ & $\begin{array}{c}130 \\
(5.2 ; 31.8 ; 42.6) \\
\end{array}$ & $\begin{array}{c}100 \\
(2.6 ; 12.5 ; 34.8) \\
\end{array}$ \\
\hline Central Europe & $\begin{array}{c}61 \\
(0.3 ; 10.3 ; 12.9)\end{array}$ & $\begin{array}{c}130 \\
(11.9 ; 10.2 ; 66.6)\end{array}$ & $\begin{array}{c}80 \\
(3.1 ; 4.8 ; 28.7)\end{array}$ \\
\hline Middle East & $\begin{array}{c}60 \\
(0.1 ; 2.6 ; 34.5) \\
\end{array}$ & $\begin{array}{c}100 \\
(7.7 ; 25 ; 59)\end{array}$ & $\begin{array}{c}70 \\
(6.9 ; 14.3 ; 37.9) \\
\end{array}$ \\
\hline Northern Africa & $\begin{array}{c}38 \\
(0 ; 1.1 ; 23.7) \\
\end{array}$ & $\begin{array}{c}60 \\
(1.2 ; 6.2 ; 41.4) \\
\end{array}$ & $\begin{array}{c}40 \\
(1 ; 4.7 ; 23.9)\end{array}$ \\
\hline Rest Central America & $\begin{array}{c}38 \\
(0.1 ; 5.1 ; 3.5)\end{array}$ & $\begin{array}{c}50 \\
(1.5 ; 13.5 ; 5.4) \\
\end{array}$ & $\begin{array}{c}40 \\
(1.2 ; 5.7 ; 4.5)\end{array}$ \\
\hline Korea & $\begin{array}{c}36 \\
(0.6 ; 5.2 ; 20.4)\end{array}$ & $\begin{array}{c}50 \\
(5.4 ; 11.8 ; 22.7)\end{array}$ & $\begin{array}{c}40 \\
(2.4 ; 8.8 ; 22.6)\end{array}$ \\
\hline Turkey & $\begin{array}{c}31 \\
(0 ; 0.3 ; 3.7) \\
\end{array}$ & $\begin{array}{c}40 \\
(0.3 ; 3.5 ; 17) \\
\end{array}$ & $\begin{array}{c}40 \\
(0.3 ; 2.5 ; 12.5) \\
\end{array}$ \\
\hline Mexico & $\begin{array}{c}29 \\
(0.1 ; 1.7 ; 11.8) \\
\end{array}$ & $\begin{array}{c}40 \\
(0.7 ; 6.5 ; 11.8) \\
\end{array}$ & $\begin{array}{c}30 \\
(0.7 ; 2.8 ; 11.8) \\
\end{array}$ \\
\hline Russia + & $\begin{array}{c}23 \\
(0.6 ; 3 ; 7.4) \\
\end{array}$ & $\begin{array}{c}40 \\
(3.8 ; 3.7 ; 19.2) \\
\end{array}$ & $\begin{array}{c}30 \\
(3.8 ; 2.6 ; 11) \\
\end{array}$ \\
\hline Japan & $\begin{array}{c}22 \\
(0 ; 6 ; 11.1)\end{array}$ & $\begin{array}{c}70 \\
(1.4 ; 18.9 ; 39.5) \\
\end{array}$ & $\begin{array}{c}30 \\
(1.2 ; 5.4 ; 16.2) \\
\end{array}$ \\
\hline Canada & $\begin{array}{c}22 \\
(0.1 ; 5.6 ; 0.2) \\
\end{array}$ & $\begin{array}{c}50 \\
(2.7 ; 26.4 ; 5.9) \\
\end{array}$ & $\begin{array}{c}30 \\
(2.2 ; 7.5 ; 5.8) \\
\end{array}$ \\
\hline Asia-Stan & $\begin{array}{c}18 \\
(0 ; 9.1 ; 1.6) \\
\end{array}$ & $\begin{array}{c}20 \\
(0.2 ; 4.4 ; 2.4) \\
\end{array}$ & $\begin{array}{c}20 \\
(0.2 ; 3.2 ; 1.7)\end{array}$ \\
\hline Oceania & $\begin{array}{c}11 \\
(0 ; 2.2 ; 3.1)\end{array}$ & $\begin{array}{c}50 \\
(0.7 ; 8.1 ; 26.7)\end{array}$ & $\begin{array}{c}20 \\
(0.7 ; 2.9 ; 11.1)\end{array}$ \\
\hline Ukraine + & $\begin{array}{c}8 \\
(0.1 ; 10.3 ; 1.8) \\
\end{array}$ & $\begin{array}{c}20 \\
(1.1 ; 4 ; 4.4) \\
\end{array}$ & $\begin{array}{c}10 \\
(0.8 ; 3.1 ; 3.3) \\
\end{array}$ \\
\hline
\end{tabular}


Table S2.9 Comparison of OC emission scenarios per world region (year 2010). Emissions are expressed in $\mathrm{Gg} \mathrm{OC/yr}$ and include all emission sectors; numbers in brackets refer to the corresponding power, industry and transport emissions.

\begin{tabular}{|c|c|c|c|}
\hline OC - 2010 & STAG_ENERGY & STAG_TECH & REF_2010 \\
\hline Globe & $\begin{array}{c}11991 \\
(24.7 ; 555.9 ; 242.5) \\
\end{array}$ & $\begin{array}{c}13800 \\
(194.6 ; 1655.6 ; 812.4) \\
\end{array}$ & $\begin{array}{c}13200 \\
(153.1 ; 1494.4 ; 384.9) \\
\end{array}$ \\
\hline China + & $\begin{array}{c}3839 \\
(9.4 ; 176.7 ; 17)\end{array}$ & $\begin{array}{c}4000 \\
(51.1 ; 880.6 ; 125.6)\end{array}$ & $\begin{array}{c}3910 \\
(49.8 ; 877.8 ; 46.6)\end{array}$ \\
\hline India + & $\begin{array}{c}2767 \\
(3.2 ; 61.1 ; 39.8)\end{array}$ & $\begin{array}{c}2840 \\
(59.3 ; 233.5 ; 52.3)\end{array}$ & $\begin{array}{c}2820 \\
(53.7 ; 231.9 ; 40.6)\end{array}$ \\
\hline Southeastern Asia & $\begin{array}{c}1161 \\
(0.5 ; 31.3 ; 24.3) \\
\end{array}$ & $\begin{array}{c}1210 \\
(3.7 ; 59.8 ; 53.7)\end{array}$ & $\begin{array}{c}1170 \\
(3.5 ; 50.1 ; 30.5)\end{array}$ \\
\hline Western Africa & $\begin{array}{c}1088 \\
(0.1 ; 15.1 ; 4)\end{array}$ & $\begin{array}{c}1090 \\
(0.2 ; 51.6 ; 4.9)\end{array}$ & $\begin{array}{c}1090 \\
(0.2 ; 51.4 ; 4.9)\end{array}$ \\
\hline Indonesia + & $\begin{array}{c}736 \\
(0.2 ; 1.8 ; 27.3)\end{array}$ & $\begin{array}{c}760 \\
(2 ; 41.7 ; 39.6)\end{array}$ & $\begin{array}{c}740 \\
(1.8 ; 41.2 ; 29.8)\end{array}$ \\
\hline Brazil & $\begin{array}{c}623 \\
(0 ; 13.6 ; 4) \\
\end{array}$ & $\begin{array}{c}690 \\
(1.8 ; 78.5 ; 35.4) \\
\end{array}$ & $\begin{array}{c}650 \\
(1.8 ; 45.6 ; 28.1) \\
\end{array}$ \\
\hline Eastern Africa & $\begin{array}{c}588 \\
(0.1 ; 2.1 ; 3.4) \\
\end{array}$ & $\begin{array}{c}590 \\
(0.4 ; 10.4 ; 5) \\
\end{array}$ & $\begin{array}{c}590 \\
(0.4 ; 10.3 ; 4.8) \\
\end{array}$ \\
\hline Southern Africa & $\begin{array}{c}454 \\
(4.6 ; 11.6 ; 11)\end{array}$ & $\begin{array}{c}450 \\
(4.7 ; 21.4 ; 10.3)\end{array}$ & $\begin{array}{c}450 \\
(4.2 ; 16.7 ; 6.5)\end{array}$ \\
\hline Rest South America & $\begin{array}{c}268 \\
(2.3 ; 4.5 ; 12.9) \\
\end{array}$ & $\begin{array}{c}290 \\
(4.1 ; 19.4 ; 26.9) \\
\end{array}$ & $\begin{array}{c}280 \\
(3.8 ; 11.1 ; 21.9) \\
\end{array}$ \\
\hline USA & $\begin{array}{c}252 \\
(0.6 ; 145.7 ; 14.6) \\
\end{array}$ & $\begin{array}{c}300 \\
(14.2 ; 81.2 ; 42.8) \\
\end{array}$ & $\begin{array}{c}280 \\
(7 ; 78.2 ; 34.5) \\
\end{array}$ \\
\hline OECD Europe & $\begin{array}{c}220 \\
(0.3 ; 28.6 ; 21.7) \\
\end{array}$ & $\begin{array}{c}470 \\
(15.8 ; 46 ; 236.1) \\
\end{array}$ & $\begin{array}{c}250 \\
(2.5 ; 19 ; 49.4) \\
\end{array}$ \\
\hline Central Europe & $\begin{array}{c}189 \\
(0.3 ; 14.8 ; 4.5)\end{array}$ & $\begin{array}{c}240 \\
(14.5 ; 17 ; 35.7)\end{array}$ & $\begin{array}{c}200 \\
(3.4 ; 7.1 ; 12.6)\end{array}$ \\
\hline Turkey & $\begin{array}{c}127 \\
(0 ; 0.4 ; 1.6)\end{array}$ & $\begin{array}{c}140 \\
(0.6 ; 9.5 ; 7.5)\end{array}$ & $\begin{array}{c}130 \\
(0.6 ; 3.4 ; 4.1)\end{array}$ \\
\hline Rest Central America & $\begin{array}{c}108 \\
(0.1 ; 3.6 ; 2.1)\end{array}$ & $\begin{array}{c}110 \\
(1.9 ; 6.5 ; 3.4)\end{array}$ & $\begin{array}{c}110 \\
(1.9 ; 4.4 ; 2.8)\end{array}$ \\
\hline Russia + & $\begin{array}{c}85 \\
(1.1 ; 6.6 ; 4.4)\end{array}$ & $\begin{array}{c}90 \\
(4.6 ; 7.7 ; 10.6)\end{array}$ & $\begin{array}{c}90 \\
(4.4 ; 5.7 ; 5.9)\end{array}$ \\
\hline Mexico & $\begin{array}{c}79 \\
(0 ; 1.7 ; 7.2)\end{array}$ & $\begin{array}{c}90 \\
(1.1 ; 10.9 ; 7.2)\end{array}$ & $\begin{array}{c}80 \\
(1 ; 2.8 ; 7.1)\end{array}$ \\
\hline Oceania & $\begin{array}{c}56 \\
(0 ; 2 ; 1.2)\end{array}$ & $\begin{array}{c}80 \\
(1.2 ; 5.8 ; 15.5)\end{array}$ & $\begin{array}{c}60 \\
(1.1 ; 2.8 ; 3.6)\end{array}$ \\
\hline Middle East & $\begin{array}{c}55 \\
(0.1 ; 1.9 ; 18.8) \\
\end{array}$ & $\begin{array}{c}70 \\
(3 ; 7.5 ; 31.7) \\
\end{array}$ & $\begin{array}{c}60 \\
(2.9 ; 6.4 ; 20.7) \\
\end{array}$ \\
\hline Northern Africa & $\begin{array}{c}49 \\
(0.1 ; 1.6 ; 14.1)\end{array}$ & $\begin{array}{c}60 \\
(0.7 ; 5.8 ; 24.6)\end{array}$ & $\begin{array}{c}50 \\
(0.7 ; 5.6 ; 14.2)\end{array}$ \\
\hline Korea & $\begin{array}{c}47 \\
(1.1 ; 4.2 ; 4)\end{array}$ & $\begin{array}{c}60 \\
(4.4 ; 16.5 ; 5.2)\end{array}$ & $\begin{array}{c}50 \\
(4 ; 7.5 ; 5.1)\end{array}$ \\
\hline Ukraine + & $\begin{array}{c}38 \\
(0.2 ; 9.5 ; 0.8)\end{array}$ & $\begin{array}{c}40 \\
(1.3 ; 6 ; 2.3)\end{array}$ & $\begin{array}{c}40 \\
(1.2 ; 2.9 ; 1.6)\end{array}$ \\
\hline Canada & $\begin{array}{c}34 \\
(0.1 ; 4.1 ; 0.2)\end{array}$ & $\begin{array}{c}50 \\
(2.4 ; 15.5 ; 4.1)\end{array}$ & $\begin{array}{c}40 \\
(2.2 ; 5.5 ; 4)\end{array}$ \\
\hline Asia-Stan & $\begin{array}{c}29 \\
(0.1 ; 9 ; 1.2) \\
\end{array}$ & $\begin{array}{c}40 \\
(0.3 ; 7.7 ; 1.7)\end{array}$ & $\begin{array}{c}30 \\
(0.3 ; 3.3 ; 1.2) \\
\end{array}$ \\
\hline Japan & $\begin{array}{c}17 \\
(0 ; 4.4 ; 2.6)\end{array}$ & $\begin{array}{c}50 \\
(1.2 ; 15 ; 30)\end{array}$ & $\begin{array}{c}20 \\
(0.6 ; 3.9 ; 4.3)\end{array}$ \\
\hline
\end{tabular}




\section{S3 Comparison of EDGAR4.3.1 reference emission scenario with other reported emissions}

In order to evaluate the consistency of the EDGARv4.3.1 reference scenario, used in this study, a comparison with other official and science based national/regional emission inventories and regional databases (e.g. HTAP_v2 and MACCity) is performed (see Figs. S3.1 and S3.2). The HTAP_v2 includes official regional emission data provided by the Environmental Protection Agency (EPA)'s for USA, EPA and Environment Canada's for Canada, the European Monitoring and Evaluation Programme (EMEP) and Netherlands Organisation for Applied Scientific Research (TNO)'s for Europe, and the Model Inter-comparison Study for Asia (MICS-Asia III)'s for China, India and other Asian countries (Janssens-Maenhout, et al., 2015). In the following, we show the good agreement obtained for $\mathrm{SO}_{2}$ and $\mathrm{BC}$, but similar results are found for all gaseous and particulate matter pollutants. Here we present the comparison at regional level aggregated for all sectors, although the comparison was also performed at sector level for specific regions of interest but not presented here. Considering Europe, USA, China and India, the agreement of regional emissions between EDGARv4.3.1 and HTAP_v2 is quite good. Differences between EDGARv4.3.1 and HTAP_v2 are between $-4 \%$ and $+4 \%$ for $\mathrm{SO}_{2},-20 \%$ and $+20 \%$ for NOx, $13 \%$ and $26 \%$ for CO, $-48 \%$ and $6 \%$ for NMVOC, while larger deviations are found for PM and its components ranging from $-53 \%$ to $14 \%$. 


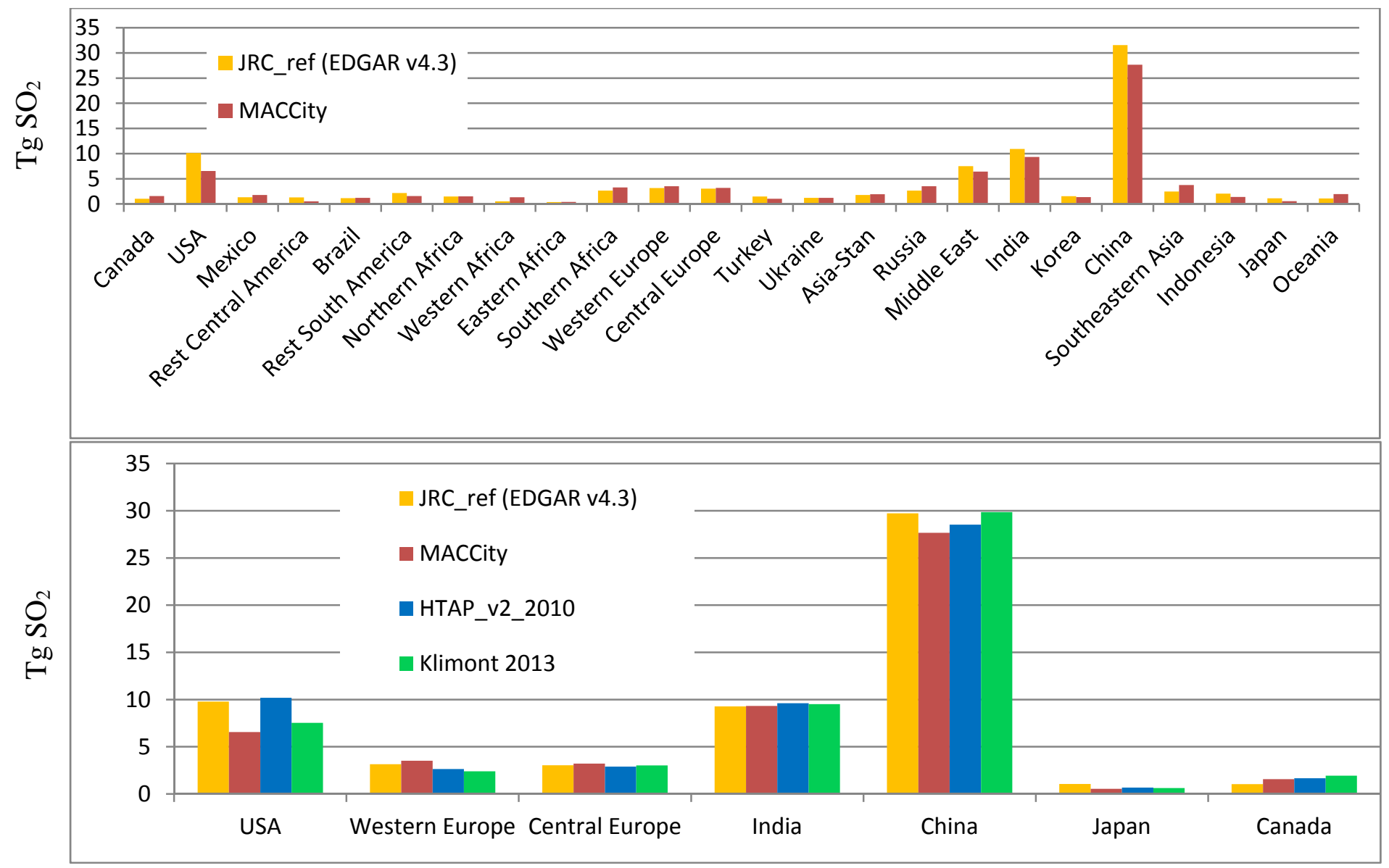

Figure S3.1 - Comparison of $2010 \mathrm{SO}_{2}$ emission data from EDGAR v4.3.1, HTAP_v2, MACCity and literature works at regional level.

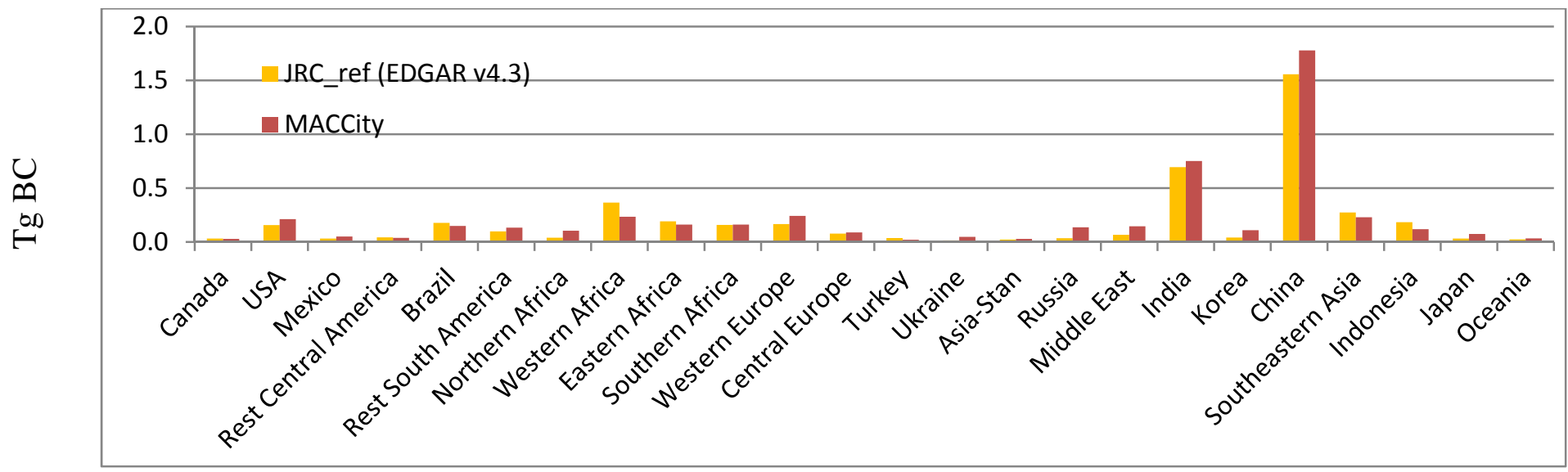

Figure S3.2 - Comparison of 2010 BC emission data from EDGAR v4.3.1 and MACCity at regional level. 
Figures S3.3, S3.4 and S3.5 show the comparison of the emission time series (1970-2010) for selected pollutants ( $\mathrm{SO}_{2}, \mathrm{NOx}, \mathrm{CO}, \mathrm{BC}$ and $\mathrm{OC}$ ) provided by the EDGARv4.3.1 and MACCity databases for groups of regions. The comparison of the two emission databases present some sense of uncertainty; however, we remark here that to some extent the two datasets cannot be considered fully independent. It is beyond the scope of this manuscript to analyze these dependencies. The relative difference between these two inventories is calculated for each year as $\mathrm{VAR}=$ (EDGARv4.3.1-MACCity)/MACCity and then averaged over time. Table S3.1 summarizes the averaged relative difference (VAR) between the emissions estimated by MACCity and EDGARv4.3.1 over time (1970-2010). At global level, the relative difference of these two sets of emission estimates is lower than considering specific regions. Groups of regions are defined as following: emerging countries include China, India, other Asian countries, Russia, Turkey and Middle East, developing countries include Central and South America and Africa, while the industrialized ones account for Oceania, USA, Canada, Easter and Western Europe. In general, the two emission inventories show similar trends over time, although specific differences can be observed in some regions. Our uncertainty evaluation is consistent with the estimates for the year 2010 provided by Janssens-Maenhout et al. (2015) using the HTAP_v2 database, where they estimate an uncertainty of $8 \%$ for $\mathrm{SO}_{2}, 4-5 \%$ for $\mathrm{NOx}$ and $\mathrm{CO}$ and larger values for BC (29\%). 

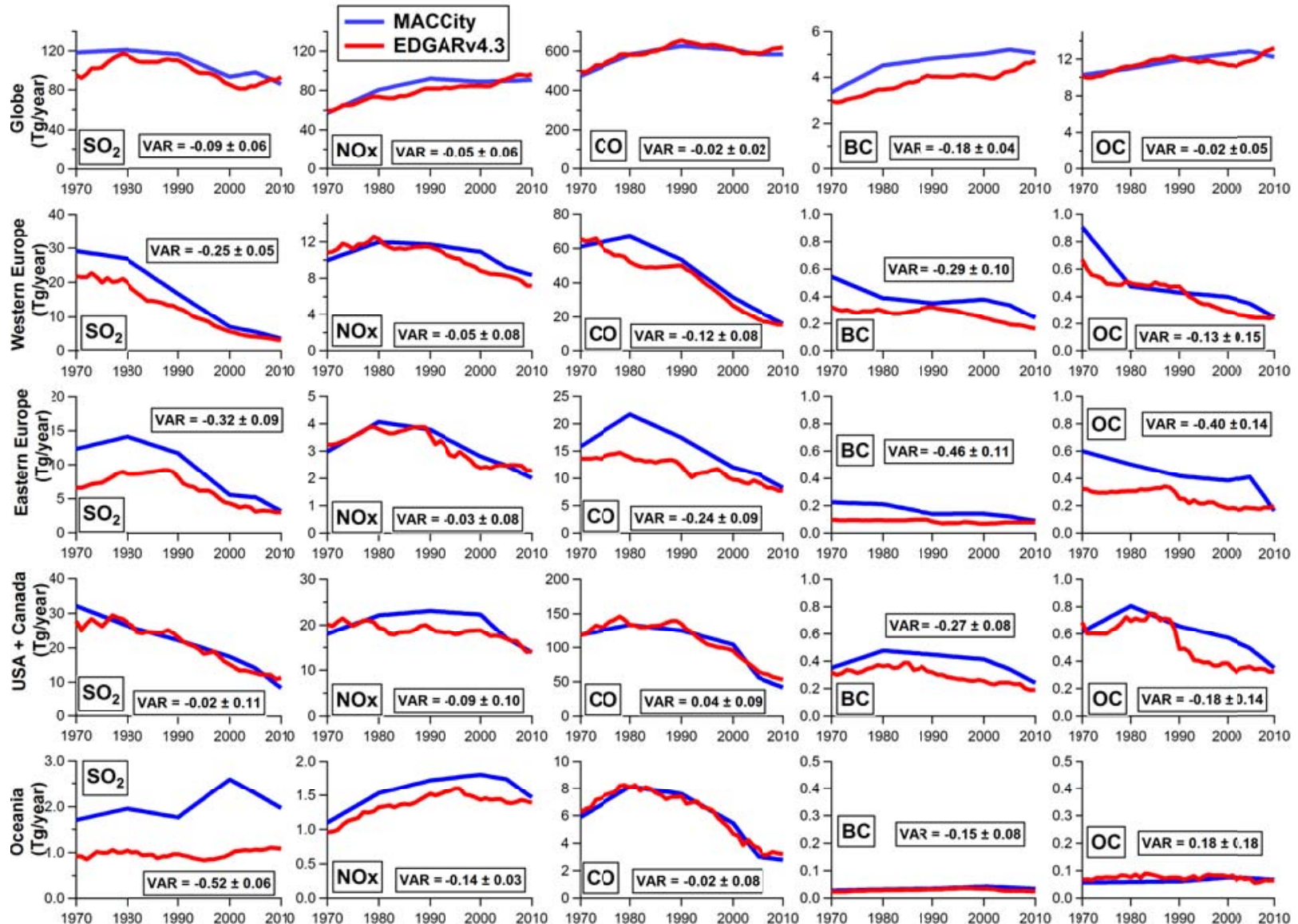

Figure S3.3 - MACCity vs EDGARv4.3.1 time series comparison (industrialized countries). 

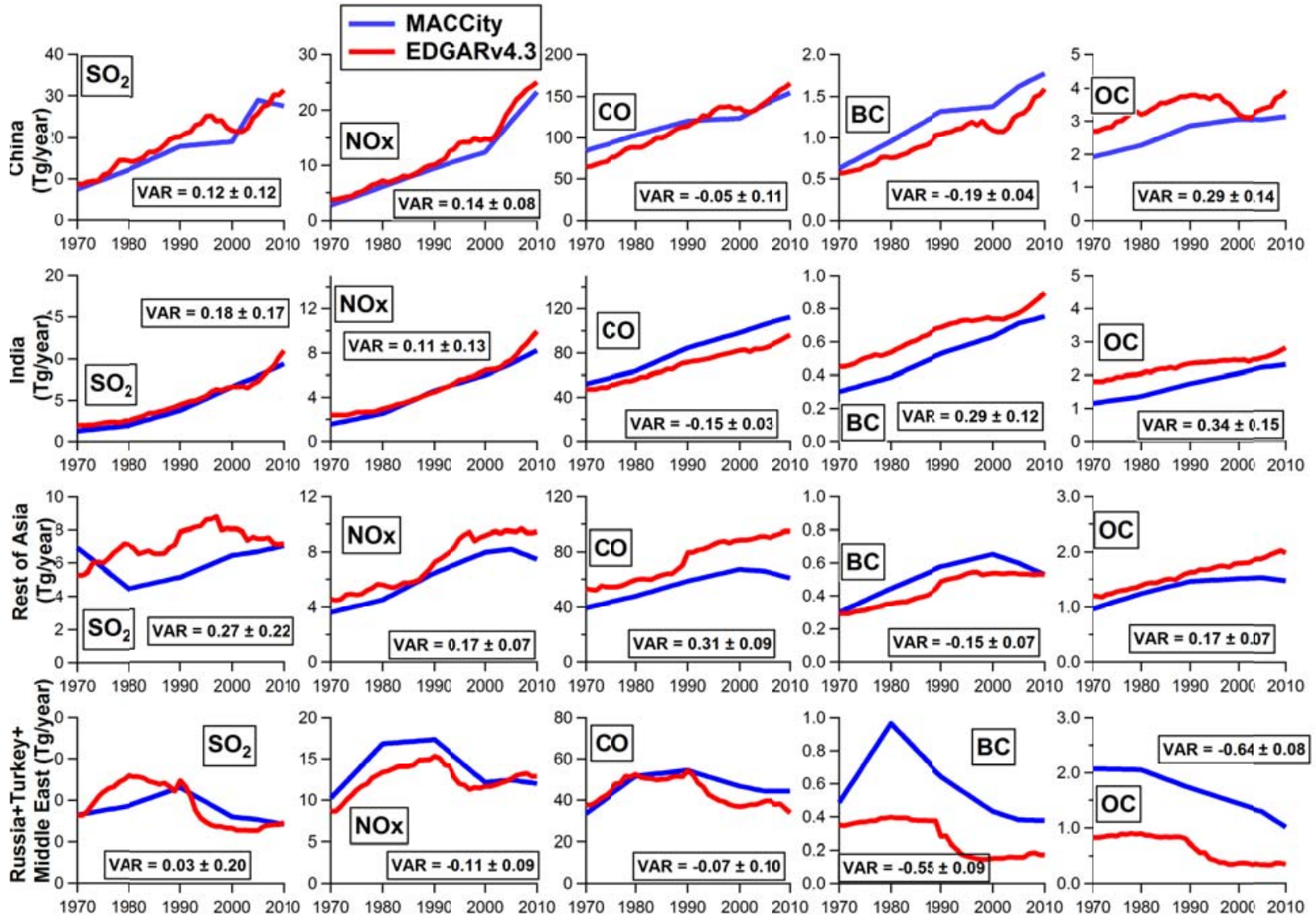

Figure S3.4 - MACCity vs EDGARv4.3.1 time series comparison (emerging countries).

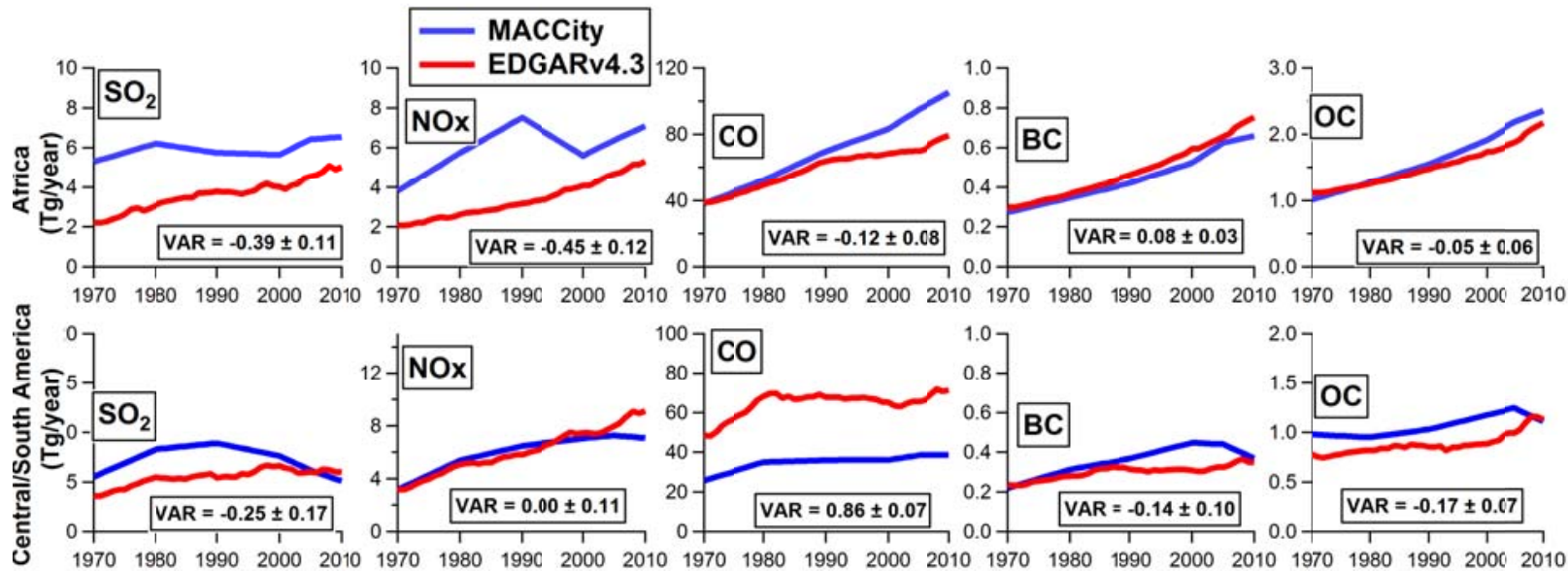

Figure S3.5 - MACCity vs EDGARv4.3.1 time series comparison (developing countries). 
Table S3.1 - Relative difference between MACCity and EDGARv4.3.1 time series for groups of regions (industrialized, emerging and developing countries). +/- 1 standard deviation based on deviations of 30 years of regionally aggregated differences.

\begin{tabular}{|l|l|l|l|l|l|}
\hline VAR \% & $\mathrm{SO}_{2}$ & $\mathrm{NOx}$ & $\mathrm{CO}$ & $\mathrm{BC}$ & $\mathrm{OC}$ \\
\hline Globe & $-\mathbf{8 . 5} \pm \mathbf{6 . 0} \%$ & $-5.2 \pm 6.0 \%$ & $1.8 \pm 2.0 \%$ & $-18.1 \pm 4.0 \%$ & $-1.6 \pm 5.0 \%$ \\
\hline Industrialized & $-27.8 \pm 20.6 \%$ & $-7.9 \pm 4.9 \%$ & $-7.6 \pm 13.0 \%$ & $-29.3 \pm 12.8 \%$ & $-13.4 \pm 23.9 \%$ \\
\hline Emerging & $14.7 \pm 10.0 \%$ & $7.7 \pm 13.0 \%$ & $\mathbf{0 . 8} \pm 20.4 \%$ & $-15.2 \pm 34.6 \%$ & $3.9 \pm 46.0 \%$ \\
\hline Developing & $-31.9 \pm 10.2 \%$ & $-22.3 \pm 31.4 \%$ & $36.8 \pm 69.3 \%$ & $-3.1 \pm 16.1 \%$ & $-10.9 \pm 8.7 \%$ \\
\hline
\end{tabular}




\section{S4 EDGARv4.3.1 emitting sector specifications and regions classification}

In this section some details about the EDGARv4.3.1 database are provided, focusing on the three emission sectors included in our scenarios (power, industry and road transport).

\section{S4.1: Power generation (ENE)}

Table S4.1.1 summarizes the processes and technologies considered in the power generation sector by the EDGARv4.3.1 database, while pollutant specific abatement measures are reported in Tables S4.1.2, 4.1.3 and 4.1.4. Numerical codes reported in Figs. S4.1.1 and S4.1.2 refer to applied abatement measures for $\mathrm{NOx}, \mathrm{PM}$ and $\mathrm{SO}_{2}$ (e.g. 000 means that no abatements are applied for the three pollutants, while increasing numbers correspond to more advanced abatement measures).

Table S4.1.1: Processes and technologies

\begin{tabular}{|l|l|l|l|}
\hline \multicolumn{4}{|c|}{ Power industry (ENE) } \\
\hline Process & Description & Technology & Description \\
\hline ENE.PEL & Public electricity production & NSF & $\begin{array}{l}\text { Non-specified } \\
\text { technology }\end{array}$ \\
\hline ENE.CHP & Public cogeneration of heat and electricity & GF0 & Grate firing \\
\hline ENE.DHE & Public district heating & PW0 & $\begin{array}{l}\text { Pulverized coal wet } \\
\text { bottom }\end{array}$ \\
\hline ENE.AEL & Autoproduced electricity & PD0 & $\begin{array}{l}\text { Pulverized coal dry } \\
\text { bottom }\end{array}$ \\
\hline ENE.AHP & $\begin{array}{l}\text { Autoproduced cogeneration of heat and } \\
\text { electricity }\end{array}$ & FB0 & Fluidized bed \\
\hline ENE.AHE & Autoproducer heat plants & BO0 & Boiler for gas/liquids \\
\hline ENE.POW & Own of electricity and heat (no emission) & IC0 & $\begin{array}{l}\text { Internal combustion } \\
\text { engine }\end{array}$ \\
\hline ENE.PUM & $\begin{array}{l}\text { pumped storage of electricity (no } \\
\text { emission) }\end{array}$ & GT0 & Gas turbine \\
\hline
\end{tabular}



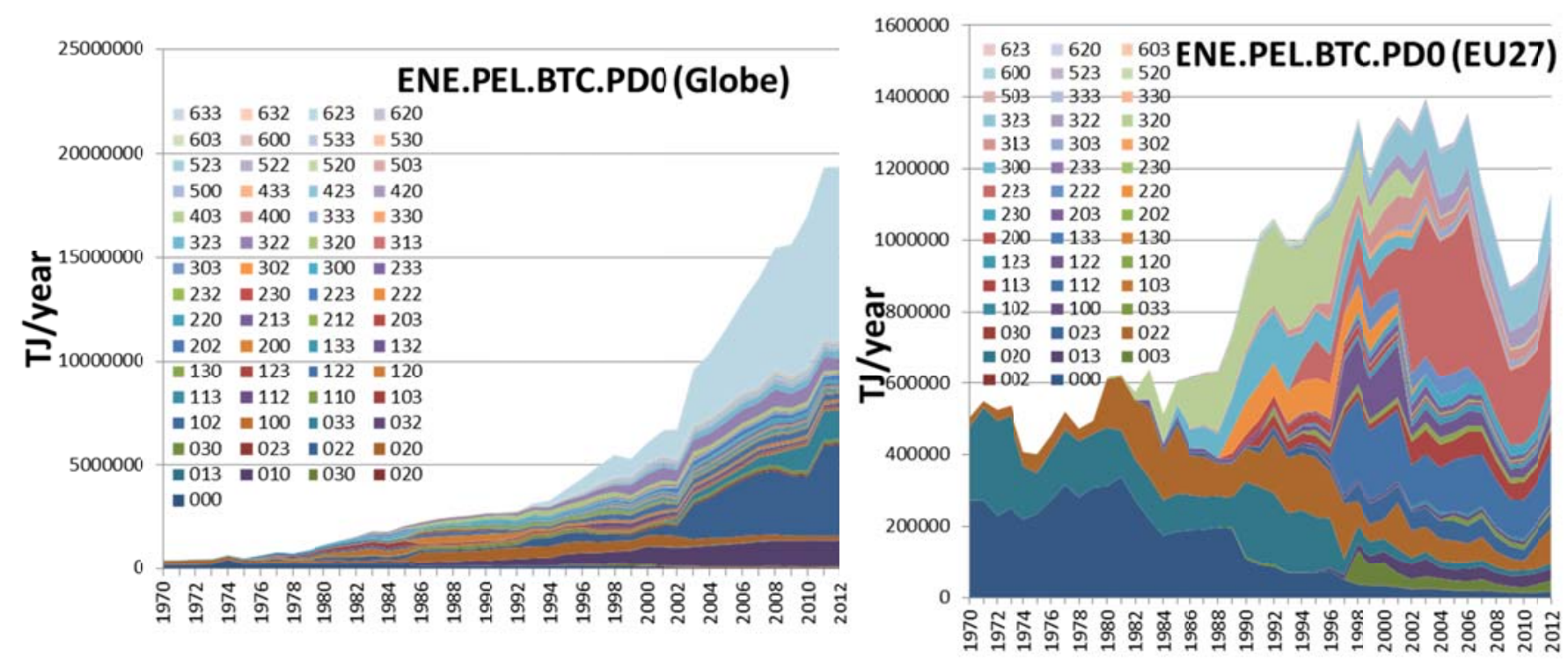

Figure S4.1.1 - Penetration of technologies applied to the power generation sector (e.g. public electricity production with bituminous coal and the technology of pulverized coal dry bottom boiler = ENE.PEL.BTC.PD0) at global and European scales.
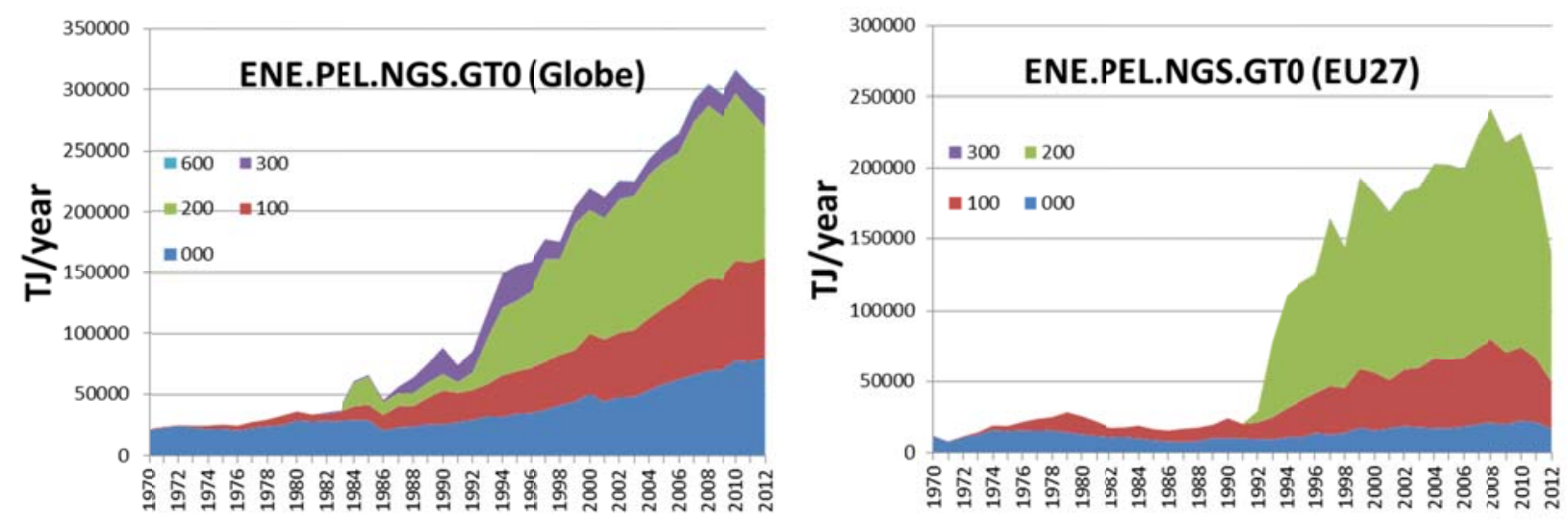

Figure S4.1.2 - Penetration of technologies applied to the power sector (e.g. public electricity production with natural gas with gas turbines $=$ ENE.PEL.NGS.GT0) at global and European scales. 


\section{Table S4.1.2: NOx abatements}

NOx abatement measures also influences $\mathrm{NH}_{3}$ emissions, increasing them by a factor of 5.5 and 11.2 for SC1/SC2 and SN1/SN2, respectively.

\begin{tabular}{|l|l|l|l|l|}
\hline Abbrev. & \multicolumn{1}{|c|}{ Description } & Reduction & EOP_code EDGARv4 & Emission reduction \\
\hline CLN & Combustion modification: low nox burners & $30 \%$ & NO1 & $30 \%$ \\
\hline CL0 & Combustion modification: low excess air & $20 \%$ & NO1 & $30 \%$ \\
\hline CAF & Combustion modification: air staging in furnace & $20 \%$ & NO1 & $30 \%$ \\
\hline CFF & Combustion modification: flue gas recirculation - in furnace & $40 \%$ & NO1 & $30 \%$ \\
\hline CR0 & Combustion modification: reduced air preheat & $20 \%$ & NO1 & $30 \%$ \\
\hline CSF & Combustion modification: fuel staging (burn or low nox & $30 \%$ & NO1 & $30 \%$ \\
\hline SC1 & Secondary selective catalytic reduction & $70 \%$ & NO2 & $60 \%$ \\
\hline SC2 & Secondary: selective catalytic reduction+ combusticn modification & $90 \%$ & NO3 & $90 \%$ \\
\hline SN1 & Secondary: selective noncatalytic reduction & $30 \%$ & NO4 & $30 \%$ \\
\hline SN2 & Secondary: selective non-catalytic reduction+ combustion modification & $50 \%$ & NO5 & $60 \%$ \\
\hline NSN & SOxNOx combined measures & $95 \%$ & NO6 & $90 \%$ \\
\hline NSF & Non-specified & $0 \%$ & NO0 & $0 \%$ \\
\hline NOC & No control & $0 \%$ & NO0 & $0 \%$ \\
\hline
\end{tabular}

\section{Table S4.1.3: SOx abatements}

SOx abatement measures have no impact on other emission components (e.g. $\mathrm{NOx}, \mathrm{NH}_{3}$, and primary PM).

\begin{tabular}{|l|l|l|l|l|} 
Abbrev. & \multicolumn{1}{|c|}{ Description } & Reduction & EOP_code EDGARv4 & Emission reduction \\
\hline SND & Non-regenerative-dry (dry FGD) & $50 \%$ & SO2 & $50 \%$ \\
\hline SNS & Non-regenerative semidry & $90 \%$ & SO3 & $90 \%$ \\
\hline SNW & Non-regenerative wet (wet FGD) & $90 \%$ & SO3 & $90 \%$ \\
\hline SRN & Regenerative & $95 \%$ & SO3 & $90 \%$ \\
\hline NSN & SOxNOx & $95 \%$ & SO3 & $90 \%$ \\
\hline NSF & Non-specified & $0 \%$ & SO0 & $0 \%$ \\
\hline NOC & No control & $0 \%$ & SO0 & $0 \%$ \\
\hline
\end{tabular}

\section{Table S4.1.4: PM abatements}

Reduction measures of $\mathrm{PM}_{2.5}$ are derived from the $\mathrm{PM}_{10}$ ones, while for $\mathrm{BC}$ and $\mathrm{OC}$ they are assumed equal to the $\mathrm{PM}_{2.5}$ ones.

\begin{tabular}{|l|l|l|l|}
\hline Abbrev. & \multicolumn{1}{|c|}{ Description } & Reduction PM10 & Reduction PM2.5 \\
\hline ESP & Electrostatic precipitator & $99.95 \%$ & $98.30 \%$ \\
\hline FBF & Fabric filter & $99.95 \%$ & $99.60 \%$ \\
\hline CYC & Cyclone & $90 \%$ & $0 \%$ \\
\hline SCR & Wet scrubber & $99.90 \%$ & $99.50 \%$ \\
\hline COM & Combination of measures & $99.95 \%$ & $98.30 \%$ \\
\hline NSF & Non specified & $0 \%$ & $0 \%$ \\
\hline NOC & No control & $0 \%$ & $0 \%$ \\
\hline
\end{tabular}




\section{S4.2: Manufacturing industry and construction (IND)}

Table S4.2.1 summarizes the processes and technologies considered in the industrial sector of the EDAGRv4.3 database.

Table S4.2.1: Processes and technologies

\begin{tabular}{|l|l|}
\hline \multicolumn{2}{|c|}{$\begin{array}{c}\text { Manufacturing industries and } \\
\text { construction (IND) }\end{array}$} \\
\hline Process & Description \\
\hline CHE & chemical \\
\hline CON & construction \\
\hline FOO & food and tobacco \\
\hline IRO & iron and steel \\
\hline MAC & machinery \\
\hline MIN & mining \\
\hline NFE & non-ferrous metals \\
\hline NMM & non-metallic minerals \\
\hline PAP & paper, pulp, print \\
\hline TEQ & transport equipment \\
\hline TEX & textiles \\
\hline WOO & wood and wood products \\
\hline INO & non-specified industry \\
\hline
\end{tabular}

\section{S4.3: Road transport (TRO.ROA)}

In this section details concerning the road transport sector as implemented in the EDGARv4.3.1 database are reported (refer to Table S4.3.1 for the processes and technologies and Table S4.3.2 for the abatement measures). In our work we only consider exhaust emissions, while break wear and re-suspension of road-dust are not taken into account. 
Table S4.3.1: Processes and technologies

\begin{tabular}{|l|l|l|l|}
\hline \multicolumn{4}{|c|}{ Road transport (TRO.ROA) } \\
\hline Fuel & Description & Technology & Description \\
\hline AVG & Aviation Gasoline & BS0 & Busses \\
\hline BDS & Biodiesel & HD0 & Heavy Duty vehicles \\
\hline BGL & Biogasoline & LD0 & Light Duty vehicles \\
\hline DIE & Gas/Diesel Oil & PC0 & Passenger cars \\
\hline OKE & Kerosene & MC0 & Motorcycles \\
\hline LPG & Liquefied Petroleum Gases (LPG) & MP0 & Mopeds (Scooters) \\
\hline MOG & Motor Gasoline & & \\
\hline NGS & Natural Gas & & \\
\hline OPR & Non-specified Petroleum Products & & \\
\hline OLB & Other liquid biofuels & & \\
\hline SBI & Primary Solid Biomass & & \\
\hline HFO & Residual Fuel Oil & & \\
\hline
\end{tabular}

Table S4.3.2: Abatement measures

\begin{tabular}{|l|l|}
\hline $\begin{array}{l}\text { Abatement } \\
\text { measures (EU) }\end{array}$ & Description \\
\hline NOC & Non controlled or conventional \\
\hline PEU & $\begin{array}{l}\text { Pre Euro standards (combined impact of } \\
\text { EU technologies before 1990) }\end{array}$ \\
\hline EU1 & Euro standard 1 \\
\hline EU2 & Euro standard 2 \\
\hline EU3 & Euro standard 3 \\
\hline EU4 & Euro standard 4 \\
\hline EU5 & Euro standard 5 \\
\hline EU6 & Euro standard 6 \\
\hline & For busses using natural gas (globally) \\
\hline PEU & Pre Euro standards \\
\hline EU1 & Euro standard 1 \\
\hline EU2 & Euro standard 2 \\
\hline EU3 & Euro standard 3 \\
\hline EEV & $\begin{array}{l}\text { Standard for Enhanced Environmental } \\
\text { Vehicles }\end{array}$ \\
\hline
\end{tabular}


American standards for passenger cars are also reported in Figs. S4.3.1 and S4.3.2, like UT1, UT2, UT3 (US Tier1-Tier3). Analogous standards are also available for heavy duty vehicles (PH1 and PH2, US Phase Tier 1 and 2 used for HDV).
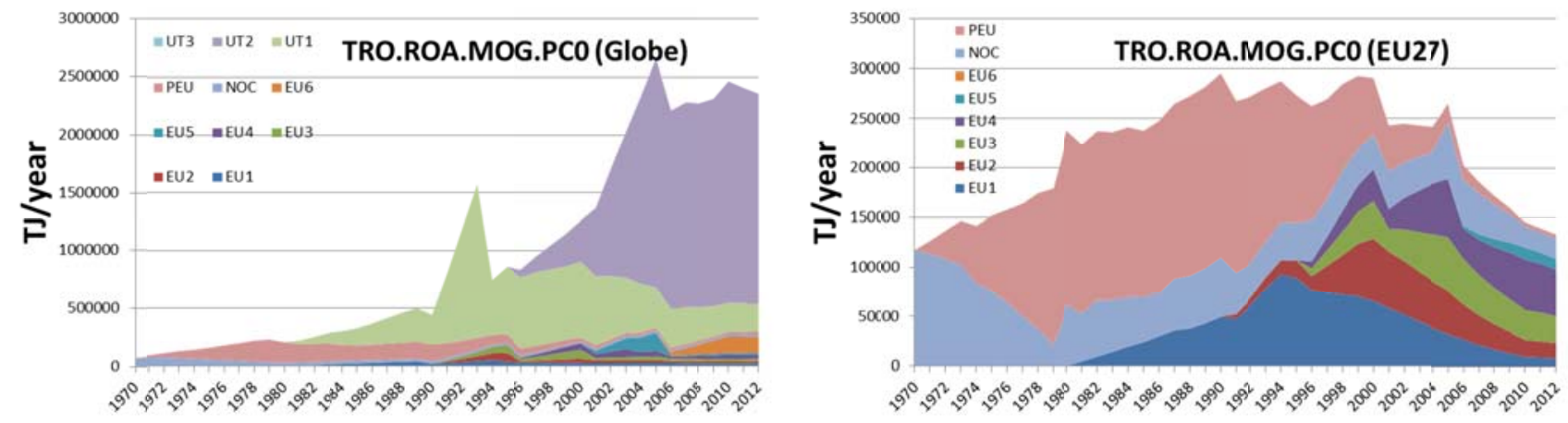

Figure S4.3.1 - Penetration of technologies applied to the road sector (e.g. motor gasoline for passenger cars = TRO.ROA.MOG.PC0) at global and European scales.
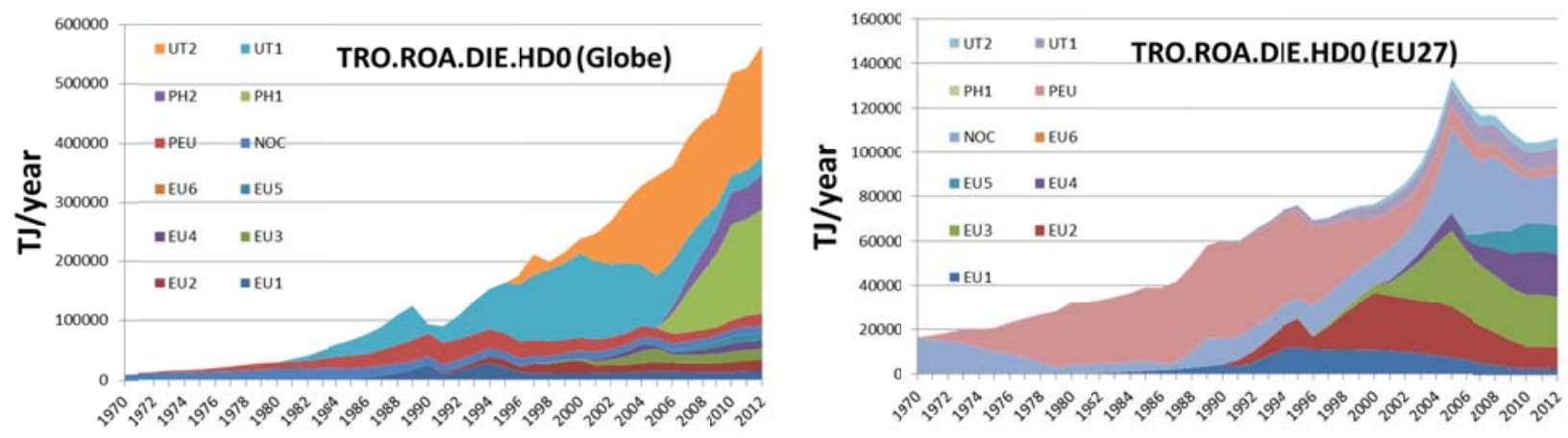

Figure S4.3.2 - Penetration of technologies applied to the road sector (e.g. diesel for heavy duty vehicles $=$ TRO.ROA.DIE.HD0) at global and European scales. 


\section{S4.4: Regions classification in EDGARv4.3.1}

In the present work emission data are often grouped by 24 emission regions (excluding Antartica), representing single geographical or political entities. However, in some cases, emissions from small countries have been added to bigger countries, like for Ukraine+ (including also Moldova and Belarus), Indonesia+ (including also Papua New Guinea), China+ (including also Hong Kong, Taiwan, Macao, Mongolia), Russia+ (including also Armenia, Georgia, Arzebaijan), India+ (including also Afghanistan, Nepal, Pakistan, etc.), Asia-Stan (including Uzbekistan, Turkmenistan, Tajikistan, Kirghizistan and Kazakhstan).
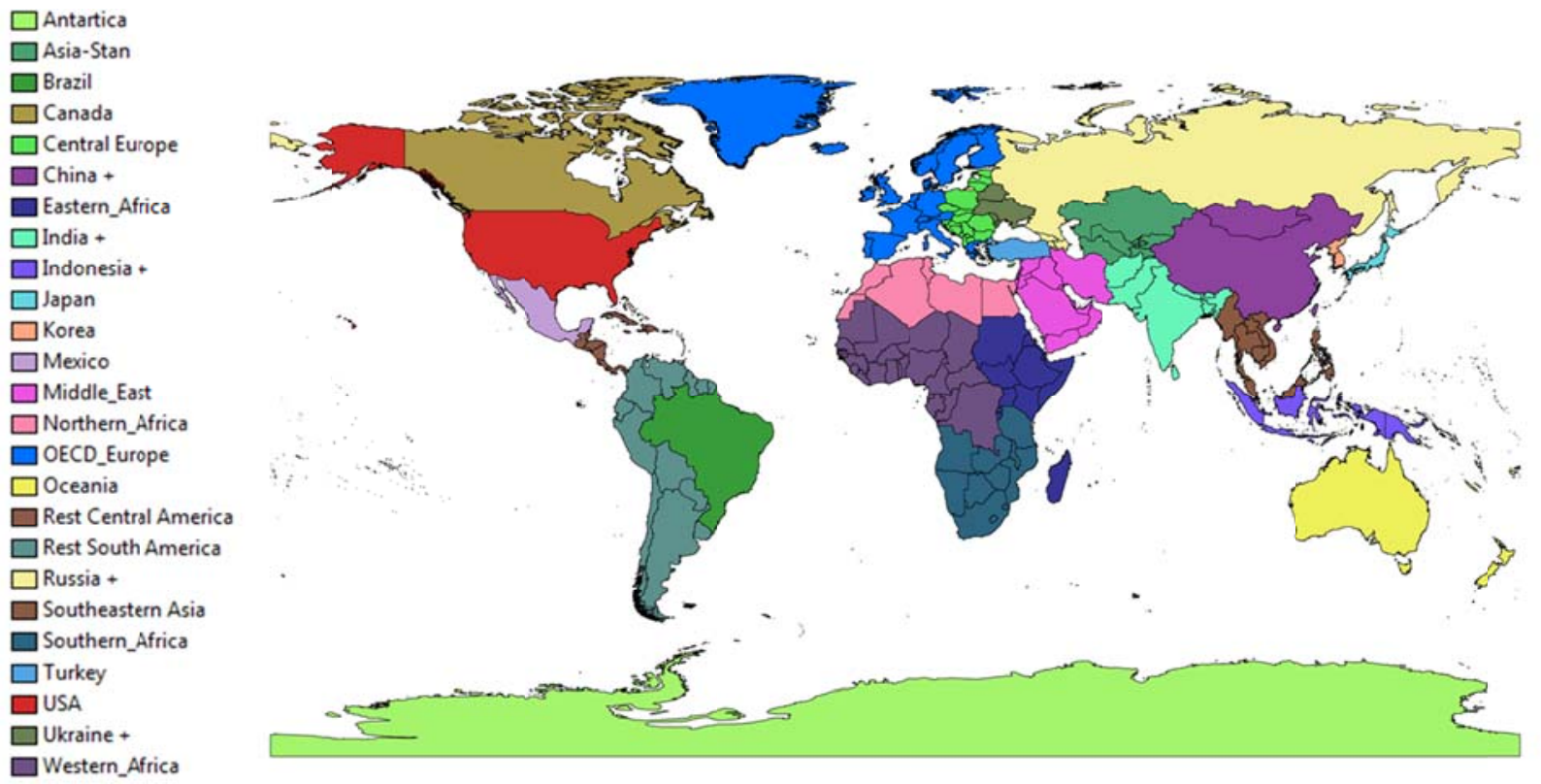

Figure S4.4.1 - Classification of world regions in the EDGARv4.3.1 database. 


\section{S5 European legislation}

The European Union (EU), as well as the extended UNECE/CLTRAP/EMEP region (http://www.ceip.at/ms/ceip_home1/ceip_home/ceip_unece/), which also includes North American and Eastern European countries, have introduced several air quality related protocols and legislation to reduce pollutant emissions in the atmosphere from anthropogenic activities (combustion processes, energy production, transportation, etc.). Table S5.1 gives an overview of historical European and international legislation pertaining to the European domain from the 1970s to 2012. European policies are classified into "air quality directives" when regulating pollutant concentrations in the air, "directives regulating air pollutants emissions from anthropogenic activities" when dealing with emission limits for specific activities, "EU standards on road vehicle emissions" and "fuel quality directives". A broader air quality regulation framework is given by international conventions which were created to promote the improvement of global air quality, like the Convention on Long-range Transboundary Air Pollution (CLRTAP) created in 1979 and later extended by several protocols such as the Gothenburg Protocol (GP) of 1999, most recently revised in 2012. 
Table S5.1 Overview of historical European legislations regulating air pollutant emissions

\begin{tabular}{|c|c|c|c|c|c|c|c|}
\hline & Policy/Pollutant & PM & SOx & NOx & $\mathrm{CO}$ & heavy metals & VOCs \\
\hline \multirow{9}{*}{ Air quality directives } & 70/220/EEC & $\mathrm{x}$ & $\mathrm{x}$ & $\mathrm{x}$ & & & \\
\hline & 80/779/EEC & $\mathrm{x}$ & $\mathrm{x}$ & $\mathrm{x}$ & & & \\
\hline & 85/203/EEC & $\mathrm{x}$ & $\mathrm{x}$ & $\mathrm{x}$ & & & \\
\hline & 96/62/EC & $\mathrm{x}$ & $\mathrm{x}$ & $\mathrm{x}$ & $\mathrm{x}$ & $\mathrm{x}$ & $\mathrm{x}$ \\
\hline & 1999/30/EC & $\mathrm{x}$ & $\mathrm{x}$ & $\mathrm{x}$ & & $\mathrm{x}$ & \\
\hline & 99/13/EC & & & & & & $\mathrm{x}$ \\
\hline & 2004/107/EC & & & & & $\mathrm{x}$ & \\
\hline & CAFE directive & $\mathrm{x}$ & $\mathrm{x}$ & $\mathrm{x}$ & & & $\mathrm{x}$ \\
\hline & 2008/50/EC & $\mathrm{x}$ & $\mathrm{x}$ & $\mathrm{x}$ & $\mathrm{x}$ & $\mathrm{x}$ & $\mathrm{x}$ \\
\hline \multirow{3}{*}{$\begin{array}{l}\text { Directives regulating air } \\
\text { pollutant emissions from } \\
\text { anthropogenic activities }\end{array}$} & $\begin{array}{l}\text { 2000/76/EC } \\
\text { (waste incineration) }\end{array}$ & $\mathrm{x}$ & $\mathrm{x}$ & $\mathrm{x}$ & & & $\mathrm{x}$ \\
\hline & \begin{tabular}{|l|} 
2001/81/EC \\
and 2010 revision \\
(national emission \\
ceilings) \\
\end{tabular} & & $\mathrm{x}$ & $\mathrm{x}$ & & & $\mathrm{x}$ \\
\hline & $\begin{array}{l}\text { 2010/75/EU } \\
\text { (industry) }\end{array}$ & $\mathrm{x}$ & $\mathrm{x}$ & $\mathrm{x}$ & $\mathrm{x}$ & & $\mathrm{x}$ \\
\hline \multirow{3}{*}{$\begin{array}{l}\text { EU standards on road } \\
\text { vehicle emissions }\end{array}$} & 94/63/EC & $\mathrm{x}$ & & $\mathrm{x}$ & $\mathrm{x}$ & & $\mathrm{x}$ \\
\hline & 1999/13/EC & $\mathrm{x}$ & & $\mathrm{x}$ & $\mathrm{x}$ & & $\mathrm{x}$ \\
\hline & 2009/126/EC & $\mathrm{x}$ & & $\mathrm{x}$ & $\mathrm{x}$ & & $\mathrm{x}$ \\
\hline \multirow{5}{*}{ Fuel quality directives } & 93/12/EC & & $\mathrm{x}$ & & & & \\
\hline & 98/70/EC & & $\mathrm{x}$ & & & & \\
\hline & 1999/32/EC & & $\mathrm{x}$ & & & & \\
\hline & 2003/17/EC & & $\mathrm{x}$ & & & $\mathrm{x}$ & $\mathrm{x}$ \\
\hline & 2009/30/EC & & $\mathrm{x}$ & & & & \\
\hline \multirow{3}{*}{ International conventions } & $\begin{array}{l}\text { CLRTAP (1979, } \\
1987 / 94 \text { and } \\
1997 / 98) \\
\end{array}$ & $\mathrm{x}$ & $\mathrm{x}$ & $\mathrm{x}$ & $\mathrm{X}$ & $\mathrm{x}$ & $\mathrm{x}$ \\
\hline & \begin{tabular}{|l|} 
Gothenburg \\
protocol (1999 and \\
2012) \\
\end{tabular} & $\mathrm{x}$ & & $\mathrm{x}$ & $\mathrm{X}$ & & $\mathrm{x}$ \\
\hline & \begin{tabular}{|l} 
IPPC directive \\
(2008/1/EC)
\end{tabular} & $\mathrm{x}$ & & & & & \\
\hline
\end{tabular}

Depending on the considered regulation, emission limits can be defined as sector specific annual emissions for selected pollutants, as total annual emissions per country (refer to 2010/75 EU), and as concentrations at the stack or in ambient air. 
Table S5.2 Implementation of European standards on vehicle emissions with PM limits $(\mathrm{mg} / \mathrm{km})$ over time. PEU refers to prior-to-Euro norms. Numerical values represent the limit value in [mg/km]. Note that Mopeds and motorcycles, using petrol, are not subject to PM limits.

\begin{tabular}{|c|c|c|c|c|c|c|c|c|c|c|c|c|c|c|c|c|c|c|c|c|c|}
\hline $\begin{array}{l}\text { Standard PM } \\
\text { limit in } \\
\mathrm{mg} / \mathrm{km}\end{array}$ & 1990 & 1991 & 1992 & 1993 & 1994 & 1995 & 1996 & 1997 & 1998 & 1999 & 2000 & 2001 & 2002 & 2003 & 32004 & 2005 & 2006 & 2007 & 2008 & 2009 & 2010 \\
\hline Busses & PEU & PEU & \begin{tabular}{|c|} 
EU1 \\
250 \\
\end{tabular} & \begin{tabular}{|c|} 
EU1 \\
250 \\
\end{tabular} & \begin{tabular}{|c|} 
EU1 \\
250 \\
\end{tabular} & \begin{tabular}{|c|} 
EU1 \\
250 \\
\end{tabular} & \begin{tabular}{|c|} 
EU2 \\
170 \\
\end{tabular} & \begin{tabular}{|c|} 
EU2 \\
170 \\
\end{tabular} & \begin{tabular}{|c|} 
EU2 \\
170 \\
\end{tabular} & \begin{tabular}{|c|} 
EU2 \\
170 \\
\end{tabular} & \begin{tabular}{|c|} 
EU3 \\
100 \\
\end{tabular} & \begin{tabular}{|c|} 
EU3 \\
100 \\
\end{tabular} & \begin{tabular}{|c|} 
EU3 \\
100 \\
\end{tabular} & $\begin{array}{c}\text { EU3 } \\
100 \\
\end{array}$ & \begin{tabular}{|c|} 
EU3 \\
100 \\
\end{tabular} & \begin{tabular}{|r|} 
EU4 \\
60 \\
\end{tabular} & $\begin{array}{r}\text { EU4 } \\
60 \\
\end{array}$ & \begin{tabular}{|r|} 
EU4 \\
60 \\
\end{tabular} & \begin{tabular}{|r|} 
EU4 \\
$\quad 60$ \\
\end{tabular} & $\begin{array}{r}\text { EU5 } \\
5\end{array}$ & EUS \\
\hline Heav & PEU & PEU & \begin{tabular}{|c|} 
EU1 \\
250 \\
\end{tabular} & \begin{tabular}{|c|} 
EU1 \\
250 \\
\end{tabular} & \begin{tabular}{|c|} 
EU1 \\
250 \\
\end{tabular} & \begin{tabular}{|c|} 
EU1 \\
250 \\
\end{tabular} & \begin{tabular}{|c|} 
EU2 \\
170 \\
\end{tabular} & \begin{tabular}{|c|} 
EU2 \\
170 \\
\end{tabular} & \begin{tabular}{|c|} 
EU2 \\
170 \\
\end{tabular} & \begin{tabular}{|c|} 
EU2 \\
170 \\
\end{tabular} & \begin{tabular}{|c|} 
EU3 \\
100 \\
\end{tabular} & \begin{tabular}{|c|} 
EU3 \\
100 \\
\end{tabular} & \begin{tabular}{|c|} 
EU3 \\
100 \\
\end{tabular} & $\begin{array}{c}\text { EU3 } \\
100 \\
\end{array}$ & $\begin{array}{c}\text { EU3 } \\
100 \\
\end{array}$ & \begin{tabular}{|c|} 
EU4 \\
60 \\
\end{tabular} & \begin{tabular}{|l|} 
EU4 \\
60 \\
\end{tabular} & \begin{tabular}{|r|} 
EU4 \\
60 \\
\end{tabular} & \begin{tabular}{|c|} 
EU4 \\
60 \\
\end{tabular} & $\begin{array}{r}\text { EU5 } \\
5\end{array}$ & EUS \\
\hline Light duty & PEU & PEU & \begin{tabular}{|c|} 
EU1 \\
190 \\
\end{tabular} & \begin{tabular}{|c|} 
EU1 \\
190 \\
\end{tabular} & \begin{tabular}{|c|} 
EU1 \\
190 \\
\end{tabular} & \begin{tabular}{|c|} 
EU1 \\
190 \\
\end{tabular} & \begin{tabular}{|c|} 
EU2 \\
120 \\
\end{tabular} & \begin{tabular}{|c|} 
EU2 \\
120 \\
\end{tabular} & \begin{tabular}{|c|} 
EU2 \\
120 \\
\end{tabular} & \begin{tabular}{|c|} 
EU2 \\
120 \\
\end{tabular} & \begin{tabular}{|c|} 
EU3 \\
70 \\
\end{tabular} & \begin{tabular}{|c|} 
EU3 \\
70 \\
\end{tabular} & \begin{tabular}{|c|} 
EU3 \\
70 \\
\end{tabular} & $\begin{array}{r}\text { EU3 } \\
70 \\
\end{array}$ & \begin{tabular}{|r} 
EU3 \\
$\quad 70$ \\
\end{tabular} & \begin{tabular}{|c|} 
EU4 \\
40 \\
\end{tabular} & \begin{tabular}{|r|} 
EU4 \\
40 \\
\end{tabular} & & \begin{tabular}{|c|} 
EU4 \\
40 \\
\end{tabular} & $\begin{array}{r}\text { EU5 } \\
5 \\
\end{array}$ & EUS \\
\hline Passenger car & PEU & PEU & \begin{tabular}{|c|} 
EU1 \\
140 \\
\end{tabular} & \begin{tabular}{|c|} 
EU1 \\
140 \\
\end{tabular} & \begin{tabular}{|c|} 
EU1 \\
140 \\
\end{tabular} & \begin{tabular}{|c|} 
EU1 \\
140 \\
\end{tabular} & \begin{tabular}{|c|} 
EU2 \\
80
\end{tabular} & \begin{tabular}{|c|} 
EU2 \\
80 \\
\end{tabular} & \begin{tabular}{|c|} 
EU2 \\
80 \\
\end{tabular} & \begin{tabular}{|c|} 
EU3 \\
50 \\
\end{tabular} & \begin{tabular}{|r|} 
EU3 \\
50 \\
\end{tabular} & \begin{tabular}{|c|} 
EU3 \\
50 \\
\end{tabular} & \begin{tabular}{|r|} 
EU3 \\
50 \\
\end{tabular} & $\begin{array}{r}\text { EU3 } \\
50 \\
\end{array}$ & \begin{tabular}{|l} 
EU3 \\
50 \\
\end{tabular} & \begin{tabular}{|c|} 
EU4 \\
25 \\
\end{tabular} & \begin{tabular}{|c|} 
EU4 \\
25 \\
\end{tabular} & \begin{tabular}{|c|} 
EU4 \\
25 \\
\end{tabular} & \begin{tabular}{|c|} 
EU4 \\
25 \\
\end{tabular} & $\begin{array}{r}\text { EU5 } \\
5 \\
\end{array}$ & EUS \\
\hline $\begin{array}{l}\text { Moped/ } \\
\text { Motorcycle }\end{array}$ & PEU & PEU & PEU & PEU & PEU & PEU & PEU & PEU & PEU & EU1 & EU1 & EU1 & EU2 & EU2 & EU2 & EU2 & EU3 & EU3 & EU3 & EU3 & EU3 \\
\hline
\end{tabular}


S6 TM5-FASST results: impacts on concentrations, health and crops
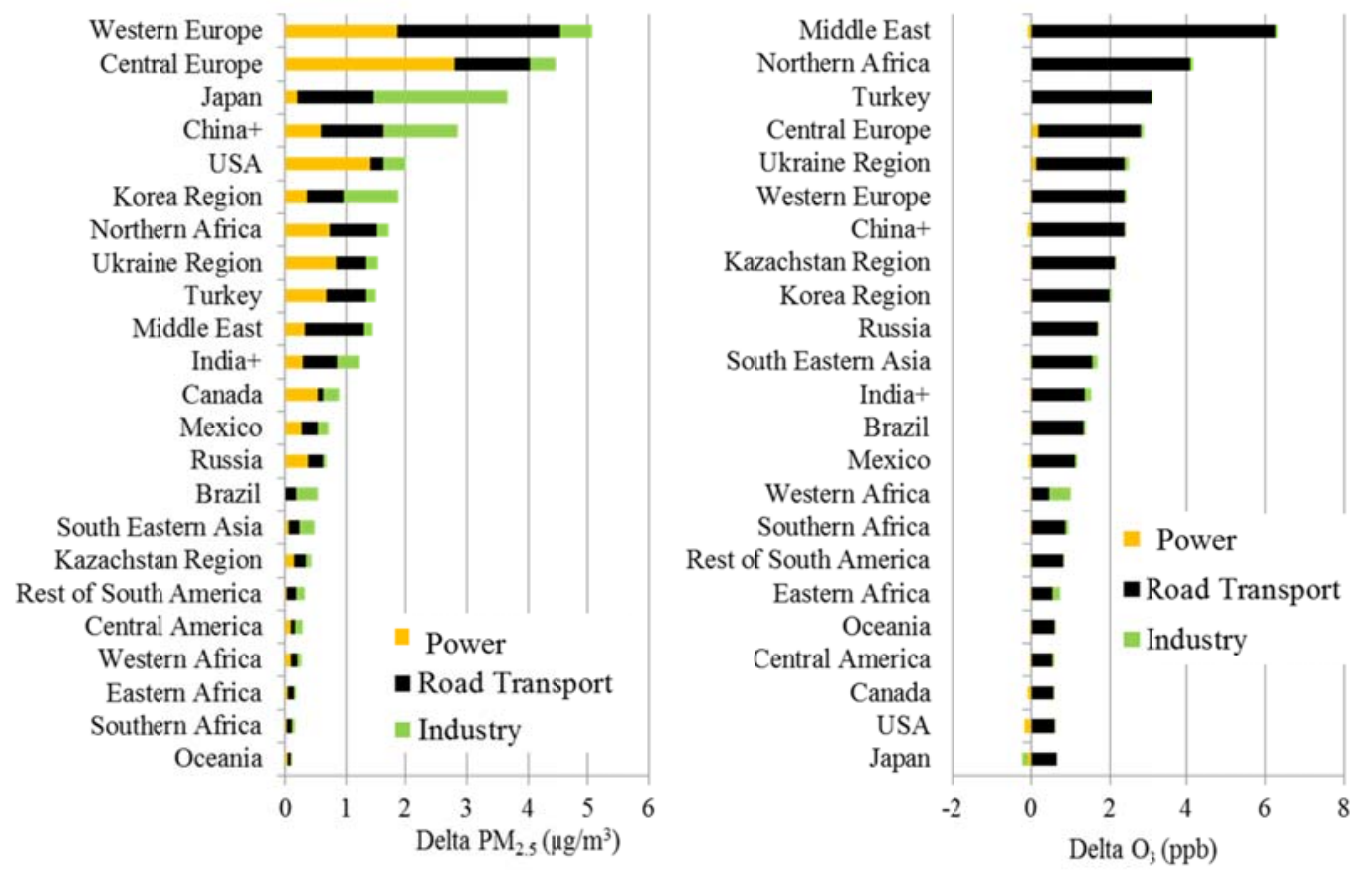

Figure S6.1 Change in $\mathrm{PM}_{2.5}$ and $\mathrm{O}_{3}$ modeled concentrations comparing STAG_TECH with the reference scenario (REF) for the year 2010.

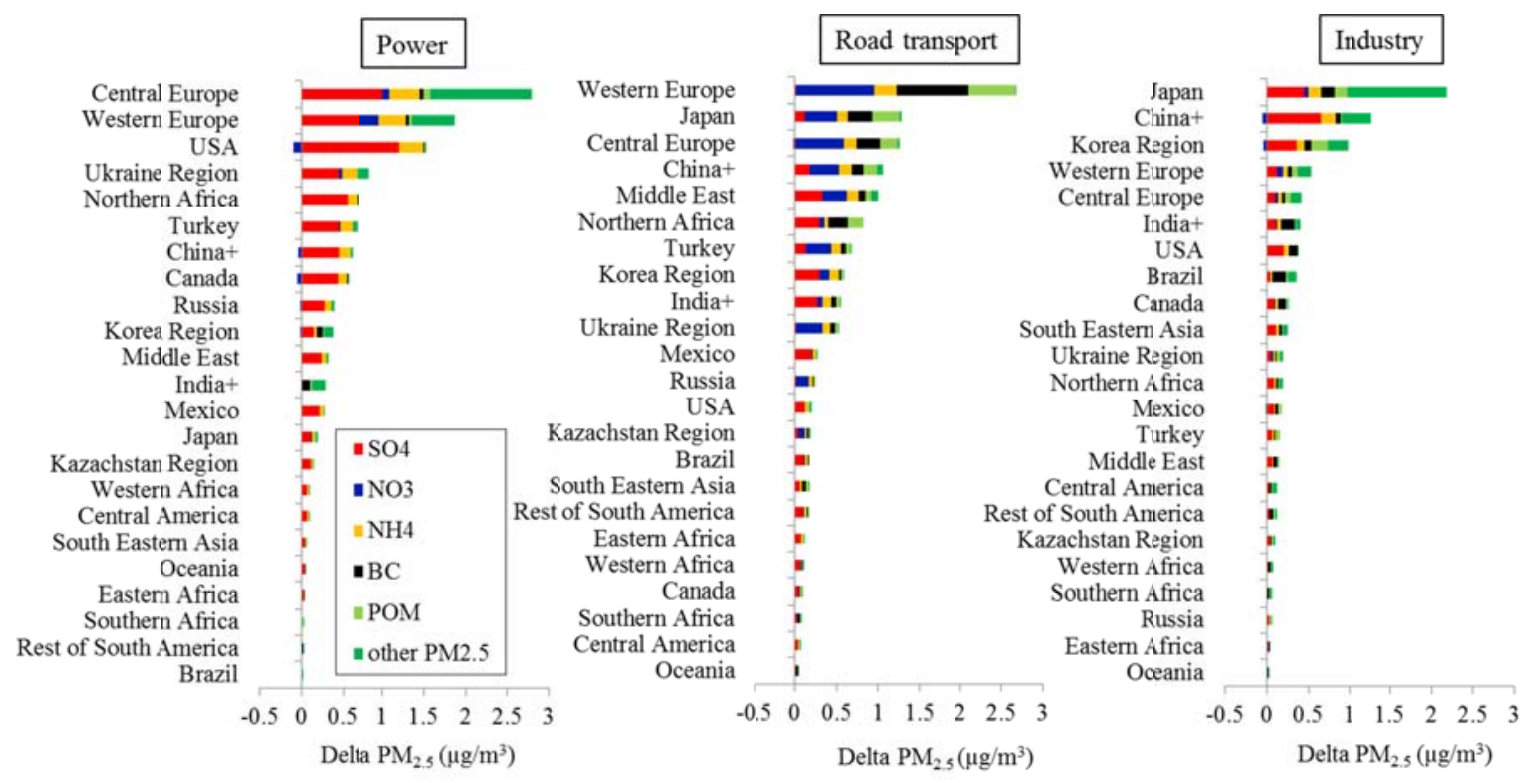

Figure S6.2 Change in regional $\mathrm{PM}_{2.5}$ chemical composition and concentration comparing STAG_TECH and REF scenarios. The comparison between the power generation, road transport and manufacturing industry sectors is reported for the year 2010. 


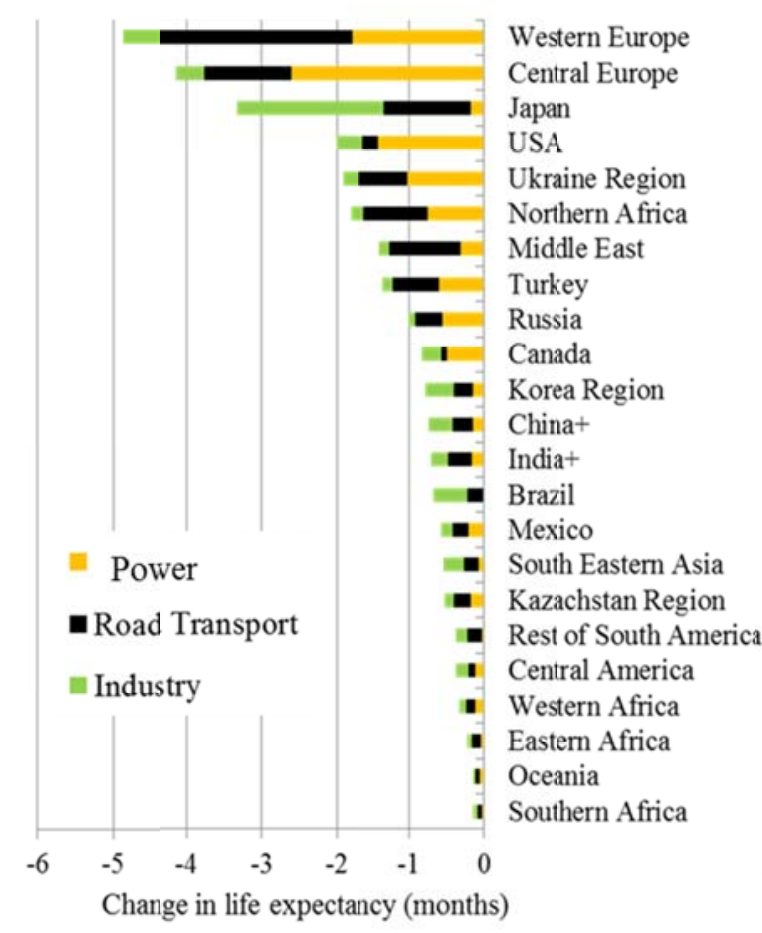

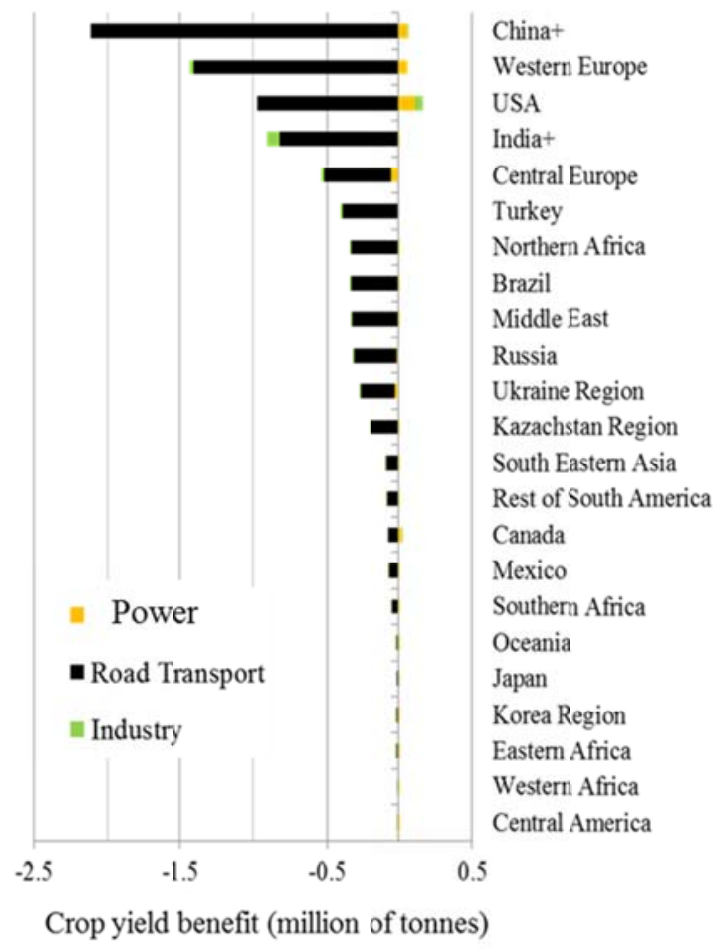

Figure S6.3 Impacts of $\mathbf{P M}_{2.5}$ and $\mathrm{O}_{3}$ concentrations on human health and crop yields. Changes in life expectancy and crop yield are obtained comparing the STAG_TECH scenario and the reference case (REF) for the year 2010. 


\section{References}

70/220/EEC: Council Directive of 20 March 1970 on the approximation of the laws of the Member States relating to measures to be taken against air pollution by gases from positive ignition engines of motor vehicles, 1970.

80/779/EEC: Council Directive 80/779/EEC of 15 July 1980 on air quality limit values and guide values for sulfur dioxide and suspended particulates, as last amended by Directive 89/427/EEC, 1980.

85/203/EEC: Council Directive 85/203/EEC of 07 March 1985 on air quality standards for nitrogen dioxide, as last amended by Council Directive 85/580/EEC, 1985.

93/12/EEC: Council Directive 93/12/EEC of 23 March 1993 relating to the sulfur content of certain liquid fuels, 1993.

96/62/EC: Council Directive 96/62/EC on ambient air quality assessment and management (Air Quality Framework Directive), 1996.

98/70/EC: Directive 98/70/EC of the European Parliament and of the Council of 13 October 1998 relating to the quality of petrol and diesel fuels and amending Council Directive 93/12/EEC, 1998.

1999/13/EC: Council Directive 1999/13/EC of 11 March 1999 on the limitation of emissions of volatile organic compounds due to the use of organic solvents in certain activities and installations, 1999.

99/30/EC: Council Directive 1999/30/EC of 22 April 1999 relating to limit values for sulfur dioxide, nitrogen dioxide and oxides of nitrogen, particulate matter and lead in ambient air, 1999.

1999/32/EC: Directive 1999/32/EC on reduction of sulfur content of certain liquid fuels, 1999.

2000/76/EC: Directive 2000/76/EC of the European Parliament and of the Council of 04 December 2000 on the incineration of waste, 2000.

2001/80/EC: Directive 2001/80/EC on the limitation of emissions of certain pollutants into the air from Large Combustion Plants, 2001.

2001/81/EC: Directive 2001/81/EC on national emissions ceilings for certain atmospheric pollutants, 2001.

2003/17/EC: Directive 2003/17/EC of the European Parliament and of the Council of 03 March 2003 amending Directive 98/70/EC relating to the quality of petrol and diesel fuels, 2003.

2004/107/EC: Directive 2004/107/EC of the European Parliament and of the Council relating to arsenic, cadmium, mercury, nickel and polycyclic aromatic hydrocarbons in ambient air (Fourth Daughter Directive), 2004.

2008/50/EC: Directive 2008/50/EC on ambient air quality and cleaner air for Europe, 2008. 
2009/30/EC: Directive 2009/30/EC of the European Parliament and of the Council of 23 April 2009 amending Directive 98/70/EC as regards the specification of petrol, diesel and gasoil and introducing a mechanism to monitor and reduce greenhouse gas emissions and amending Council Directive, 2009.

2010/75/EU: Directive 2010/75/EU of the European Parliament and of the Council of 24 November 2010 on industrial emissions (integrated pollution prevention and control), 2010.

Janssens-Maenhout, G., Crippa, M., Guizzardi, D., Dentener, F., Muntean, M., Pouliot, G., Keating, T., Zhang, Q., Kurokawa, J., Wankmüller, R., Denier van der Gon, H., Klimont, Z., and and Frost, G.: HTAP_v2: a mosaic of regional and global emission gridmaps for 2008 and 2010 to study hemispheric transport of air pollution, Atmos. Chem. Phys., 15, 1-21, 2015. 